\title{
Poliploidía En Moluscos De Importancia Comercial. A Review
}

\section{Rosalío Maldonado-Amparo Dr.}

Centro de Estudios Tecnológicos del Mar 03, Carretera al Varadero

Nacional, Sector Playitas, A.P. 742, Guaymas, Sonora México

Cesar Arturo Ruiz Verdugo, Dr.

Programa de Posgrado en Ciencias Marinas y Costeras de la Universidad Autónoma de Baja California-UABCS-, Carretera al Sur Km 5.5, A.P. 19B, La Paz, B.C.S. C.P. 23080

\section{Ana María Ibarra, Dr.}

Aquacultural Genetic Laboratory, Centro de Investigaciones Biológicas del Noroeste, S.C. A.P. 128, La Paz B.C.S. México 23000.

\section{Edgar Omar Rueda-Puente, Dr.}

Carmen Lizette Del Toro Sánchez, Dr.

Francisco Rodríguez Félix, Dr.

Universidad de Sonora, Departamento de Agricultura y Ganadería, Carretera a Bahía de Kino km. 21. Hermosillo, Sonora, México. C. P. 23000.

\begin{abstract}
Polyploidy is a biotechnology that in the last decade had provided with an improvement method for mollusks in aquaculture. Triploids have three sets of chromosomes rather than the two carried by diploids. The third set has an effect on chromosomes synapsis, producing sterility during reproduction. That sterility is the most accepted cause for the better growth seen in triploids. Artificial induction to triploidy has some problems, which can be solved by the existence of tetraploids, which could be mated to diploids to produce biological triploids. Control strategies based on prevention of disease and methods that slow the spread of such diseases. Therefore, proper management of diseases affecting the tomato crop, knowledge and understanding of the diagnosis and its infectious cycle is vital and to establish effective control measures.However, the literature is limited when it comes to auscultating information related with the poliploidía in mollusks, it's origin and the multiple biotechnologies that develop contemporary. This study was conducted to assess and meet the trend of existing research on polyploidy in commercially important shellfissh.
\end{abstract}


Keywords: Mollusks, Triploidy, tetraploid

\section{Resumen}

La poliploidía ha sido una de las biotecnologías más empleadas en la última década para el mejoramiento genético de moluscos bivalvos por ser una de las mejores alternativas a corto plazo para incrementarla producción. Los organismos triploides presentan tres juegos completos de cromosomas homólogos que afectan la sinapsis, lo cual da como resultado una esterilidad parcial o total, la cual teóricamente repercute favorablemente en el crecimiento. Sin embargo, la inducción a la triploidía presenta algunos problemas que podrían resolverse con la disponibilidad de tetraloides para producir triploides biológicos.Sin embargo, la literatura es escasa cuando se trata de auscultar información relacionada con la poliploidía en moluscos, su origen y las multiples biotecnologías que se desarrollan contemporáneamente. El presente trabajo se realizó con la finalidad de valorar y conocer la tendencia de las investigaciones sobre la Polyploidía en Moluscos de Importancia Comercial.

Palabras clave: Moluscos, Triploidía, Tetraploidía.

\section{Introducción}

Historia-origen de los primeros estudios(Triploidía en moluscos).Las primeras referencias de la obtención de moluscos triploides se remontan a 1979, cuando John G. Stanley, Stan Allen y H. Hidu evalúan la inducción a la triploidía en la ostra americana Crassostrea virginica (Stanley, 1981). Los resultados reportados por Stanley y colaboradores fueron los primeros reportes de literatura científica de triploides bivalvos viables. Con el apoyo de la NSF, el conocimiento de bivalvos triploides fue expandido. De 1983 a 1986 en la Universidad de Washington, en cooperación con Coast Oyster Company y Wescott Bay Sea Farms, los resultados obtenidos en estudios acerca de la biología y producción de ostiones triploides de Crassostrea gigas, elevaron a escalamiento de su producción a niveles comerciales (Stanley et al., 1981; Allen, 1988).

Métodos de inducción a la triploidía.La inducción artificial a la triploidía en bivalvos y gasterópodos es posible de realizar, debido a que en hembras de estos organismos los gametos femeninos liberados al medio son ovocitos primarios. Esto es, la meiósis, proceso mediante el cual ocurren las reducciónes cromosómicas, para formar gametos haploides, está detenida antes de que ocurra cualquiera de las dos reducciones cromosómicas. Al entrar el esperma y permanecer como un pronúcleo independiente del núcleo del ovocito, el proceso meiótico del ovocito es reactivado para culminar con 
la formación de ovocitos haploides después de la formación de dos cuerpos polares. La triploidía ha sido inducida por diferentes tipos de tratamientos, agrupados en "físicos" o "Químicos". Los tratamientos físicos comprenden choques de temperatura y presión hidrostática, mientras que los químicos involucran el uso de compuestos como la citocalasina-B o 6dimetilaminopurina. Dadas las características de la meiosis en estos organismos, los tratamientos pueden ser aplicados ya sea durante la meiosis I (MI) o meiosis II (MII), con fines de bloquear la formación del primer (MI) o segundo cuerpo polar (MII). Todos estos métodos buscan prevenir la división citoplásmica que tendría como consecuencia una reducción cromosómica. El resultado de inhibir la división citoplásmica es un ovocito cuyo pronúcleo femenino será diploide y la posterior "singamia" (fusión del pronúcleo femenino y masculino) con un pronúcleo masculino haploide permitirá la formación de un cigoto triploide (Beaumont y Fairbrother, 1991).

Los choques de temperatura y la presión hidrostática actúan de forma similar en evitar la división citoplasmática. La temperatura promueve que se disuelvan las fibras del huso cromático que separa los cromosomas dentro de la célula, evitando así la formación del cuerpo polar al no ocurrir la división cromosómica. La presión hidrostática inhibe la formación ó extrusión de los cuerpos polares por la misma presión ejercida sobre la pared celular del ovocito (Downing, 1987, citada por Allen et al., 1989). En el caso del uso de la temperatura, en la inducción a la triploidía en moluscos los ovocitos son sometidos a choques tanto fríos como calientes, según la especie (Yamamoto y Sugawara, 1988; Canello et al., 1992; Toro et al., 1995a; 1995b). En lo que respecta a la presión hidrostática, el método en moluscos consiste en someter los ovocitos a choques de presión hidrostática que puede variar de 6000 a 8000 psi (Allen et al., 1986).

Múltiples estudios en moluscos con ambos métodos han demostrado que los tratamientos químicos con sustancias como la citocalasina-B (CB) son los más efectivos en inducir un alto porcentaje de triploides, por lo que en la actualidad este método es el más utilizado. La CB, un compuesto originalmente desarrollado como un antibiótico, actúa inhibiendo la polimeración de la actina que produce el anillo contráctil para la formación de los cuerpos polares inhibiendo así la reducción cromosómica (Longo, 1972). La CB es hidrofóbica, por lo que para lograr que penetre en el ovocito, se disuelve en dimetilsulfóxido (DMSO) como una solución transporte. Los ovocitos fertilizados son expuestos a esa solución a concentraciones y tiempos variables. En moluscos diferentes concentraciones de CB y tiempos de aplicación han sido evaluadas para múltiples especies (Tabla 1). Los tiempos de aplicación varían dependiendo de cuál reducción cromosómica se pretende inhibir (la asociada con MI o la de MII), así como 
también depende de los tiempos exactos después de la fertilización en que cada una de esas reducciones cromosómicas ocurren. Otro químico utilizado en la inducción a la triploidía es el 6-Dimetilaminopurina, el cual actúa de forma diferente que la CB, ya que ataca la organización y depolimerización de los microtúbulos que forman el huso acromático y sobre los filamentos corticales. Esto induce al bloqueo del movimiento de los cromosomas inhibiendo la anafase y la formación de cuerpos polares (Desrosier et al., 1993; Gérard et al., 1994b; Brake et al., 2002).

Otros compuestos químicos como la cafeína y el calcio, los cuales actúan sobre la polimerización y depolimerización de microtúbulos, han sido también evaluados para la inducción a la triploidía, observando que en general su éxito es bajo (Beaumont y Fairbrother, 1991).

El grupo de Genética Acuícola del Centro de Investigaciones Biológicas del Noroeste S.C. (CIBNOR), publicó un Manual práctico para la nducción a la triploidía con Citocalacina B en el ostión Japonés Crassostrea gigas (Ibarra-Humphries et al., 2008). Recientemente Melo et al. (2015), evaluaron métodos físicos y químicos para la inducción a la triploidía en la misma especie y Suquet et al. (2016) determinaron la calidad de los gametos en el ostión triploide Crassostrea gigas.

Especies de moluscos inducidas a la triploidía utilizando citocalacina-B (CB).La triploidía ha sido inducida en abulones, almejas, mejillones, ostiones, ostras y pectínidos o escalopas (Tabla I). En haliótidos ó abulones, se ha evaluado el uso de CB en Haliotis midae, alcanzando un éxito de 70.9 \% (Stepto y Cook, 1998). Otras especies de abulones donde se ha inducido la triploidía es Haliotis rufescens, Haliotis rubra (Leach, 1814) y la especie Haliotis laevigata (Donovan) (Maldonado-Amparo et al., 2001; Liu et al., 2004; Dusten et al., 2007). En un grupo de almejas, los miídos, se evaluó en Mya arenaria la CB, obteniendo porcentajes de 75 a 100\% de triploidía (Allen et al., 1982). En mejillones ó mitílidos, como Mytilus edulis y $M$. galloprovinciallis se han obtenido porcentajes de triploidía de 38-86\% (Beaumont y Kelly, 1989; Scarpa et al., 1994). El principal grupo donde se ha trabajado más ampliamente la inducción a la triploidía es en los ostiones ó ostréidos, incluyendo a Crassostrea virginica, C. gigas, Ostrea edulis y Saccostrea commercialis, en las cuales se han alcanzado porcentajes de triploidía desde 16 hasta 100\%. Los mejores resultados en este grupo se han obtenido con concentraciónes que van de 0.5 a $1.0 \mathrm{mg} / \mathrm{L}$ de $\mathrm{CB}$, donde se han alcanzado éxitos a la triploidía de 94-100 \% (Allen y Bushek, 1992; Allen y Downing, 1986; Barber y Mann, 1991; Barber et al., 1992; Desrosiers et al., 1993; Downing y Allen, 1987; Gerard et al., 1999; Gerard et al., 1994b; Hawkins et al., 1994; Nell et al., 1994; Nell et al., 1996; Stanley et al., 1981; Stanley et al., 1984; Supan et al., 2000). El segundo grupo donde existe un gran número de trabajos reportados es en los 
pectínidosó escalopas, como Argopecten irradians, A. ventricosus, Chlamys varia, Pecten maximus; Placopecten magellanicus y Nodipecten subnodosus. Los porcentajes más elevados de triploidía los obtuvo Ruiz-Verdugo et al.(2001a), utilizando $0.5 \mathrm{mg} / \mathrm{L}$ de CB, realizando las inducciones en forma individual por familia. Se observó que no en todas se obtuvo el $100 \%$, lo que indica que el éxito a la triploidía depende no solo de la concentración y duración del tratamiento, si no de otros factores asociados a cada desove, un factor discutido por pocos autores (Baron et al., 1989; Beaumont, 1986; Jackson et al., 2002; Ruiz-Verdugo et al., 2000; Ruiz-Verdugo et al., 2001a; Maldonado-Amparo et al., 2004). En los pterídos ó ostras perleras, como Pinctada fucata martensii, se han alcanzado valores de éxito a la triploidía de 52 a 100\%, (Jiang et al., 1993; Uchimura et al., 1989; Wada et al., 1989). El tercer grupo donde se han realizado trabajos de inducción a la triploidía es en otro grupo de almejas, los venéridos, como Ruditapes philippinarum y R.decussatus, en las cuales se ha tenido un éxito de 36 a 85 \%, (Dufy y Diter, 1990; Gerard et al., 1994a; Gosling y Nolan, 1989; Nell et al., 1995; Utting y Child, 1994; Utting y Doyou, 1992) (Tabla I).

Características generales del triploide. Gametogénesis y esterilidad. La gametogénesis en moluscos triploides ha sido catalogada en dos formas, como retardada, (implicando una ovogénesis y espermatogénesis con un desarrollo atrasado pero similar a los diploides) (Eversole et al., 1996; Cox et al., 1996; Allen y Downing, 1991; Allen et al., 1986; Tabarini, 1984), $\mathrm{y}$ como arrestada (Ruiz-Verdugo et al., 2000; Kiyomoto et al., 1996; Barber y Mann, 1991).El rasgo más importante de los organismos triploides es el pobre desarrollo gonádico, el cual puede variar entre, e incluso dentro de, especies (Beaumont y Fairbrother, 1991), produciendo ya sea una esterilidad parcial (gametogénesis reducida con producción de algunos gametos) o total (gametogénesis inhibida sin producción de gametos). La esterilidad es una consecuencia de una sinapsis irregular cuando tres juegos de cromosomas homólogos ocurren en un organismo durante la profase de la meiosis I (Thorgaard, 1983; Allen, 1987a). Esta condición de esterilidad en especies de importancia comercial es importante, ya que ha sido demostrado que produce ventajas sobre los diploides, como son el presentar una mayor tasa de crecimiento, mejor calidad de la carne y mejor sabor (Allen y Downing, 1986; Allen y Downing, 1991; Beaumont y Fairbrother, 1991).

La esterilidad total no ocurre en todos los moluscos. Cuando la esterilidad no es total, la fecundidad y el potencial reproductivo de los triploides puede ser estudiado como ha sido para algunas especies, como Mya arenaria (Allen et al., 1986), Mulinia lateralis (Guo y Allen, 1994a), Crassostrea gigas (Guo y Allen, 1994b; Guo y Allen, 1997), Ruditapes philippinarum (Utting et al., 1996), Saccostrea commercialis (Cox et al., 1996) y Argopecten ventricosus (Ruiz-Verdugo et. al., 2001a; 2000). En 
estos estudios se ha observado que los organismos triploides no son completamente estériles. Por ejemplo, para Crassostrea gigas la fecundidad de un triploide se estimó que se reduce en 92 a 98 \% con relación a la fecundidad de los diploides (Guo y Allen, 1994b); en Argopecten ventricosus el triploide presenta una reducción del 82\% de la fecundidad del diploide (Ruiz-Verdugo et al., 2001a), y en Ruditapes philippinarum se estimó una reducción de fecundidad del 68\% con respecto a los diploides (Utting et al., 1996). Otras especies con esterilidad parcial son Mercenaria mercenaria y Pinctada martensii (Eversole et al., 1996; Komaru y Wada, 1990).

Por otro lado, se ha observado que algunas de las especies evaluadas para fertilidad, solo un sexo es capaz de desarrollar gametos. Por ejemplo en Argopecten ventricosus, a pesar de ser un organismo hermafrodita funcional como diploide, en triploides de un año de edad se encontró la parte masculina degenerada, desarrollándose sólo gónada femenina (Ruiz-Verdugo et al., 2000). La importancia de este resultado radica en el hecho de que uno de los problemas principales en estudios de ploidía de esta especie ha sido la inhabilidad de separar gametos de diferente sexo totalmente, ocurriendo generalmente como consecuencia la autofecundación de un cierto porcentaje de los ovocitos. Con la disponibilidad de organismos triploides que desarrollan sólo un tipo de gameto (femenino), ese problema podrá ser minimizado durante la producción de tetraploides de esta especie. Relacionado con la misma especie, existen resultados sobre el efecto de la triploidía en la estructura y abundancia de diferentes estadios de desarrollo gamético en la almeja Catarina: características ultraestructurales de la espermatogénesis en diploides y triploides (Maldonado-Amparo et al., 2002a) y un análisis comparativo de la frecuencia del tipo de ovocito diploide y triploide como indicador del arresto meiótico (MaldonadoAmparo et al., 2002b).

Condición fisiológica /Composición bioquímica de los triploides. Previo a la gametogénesis, y cuando el alimento es abundante, en los moluscos se realiza un almacenamiento de lípidos, proteínas y glucógeno, las cuales son canalizadas posteriormente para la reproducción (Barber y Blake, 1991; Sphigel et al., 1992). Debido a la condición de esterilidad parcial en los triploides, se han realizado varios estudios con diferentes especies de moluscos para evaluar el uso de las reservas energéticas para la gametogénesis en triploides versus diploides. Por ejemplo, en Crassostrea gigas se ha observado que en diploides el contenido de glucógeno disminuye constantemente durante el proceso de gametogénesis, alcanzando el nivel más bajo durante el periodo de desove, mientras que en triploides el contenido de glucógeno fue significativamente más grande durante el mismo periodo (Allen y Downing, 1986; Allen, 1987b; Akashige y Fushimi, 1991; Shpigel et al., 1992). Los mismos resultados han sido obtenidos en otras 
especies de ostión, como el ostión Australiano, Saccostrea commercialis (Nell et al., 1994). En triploides de la almeja Ruditapes philippinarum se han observado diferencias significativas en el contenido de lípidos (Shpigel y Spencer, 1996), ó de lípidos y carbohidratos (Utting et al., 1996). En pectínidos como Argopecten irradians, el contenido de glucógeno en músculo aductor de triploides es mayor que en diploides (Tabarini, 1984), mientras que en triploides de Argopecten ventricosus el contenido de carbohidratos en músculo no indicó un almacenamiento de esta forma de energía en el músculo aductor (Ruiz-Verdugo et al., 2001b) a pesar de observarse una esterilidad parcial en los triploides, así como una ventaja en crecimiento sobre los diploides. Palacios et al. (2004), realizaron la comparación de la composición bioquímica e hipertrofia del musculo aduptor asociado con el ciclo reproductivo de diploides y triploides de Argopecten ventricosus. Racotta et al. (2008), para la almeja mano de león Nodipecten subnodosus, compararon la composición bioquímica en grupos de diferentes niveles de ploidía para soportar la hipótesis fisiológica de la ausencia de ventaja en crecimiento en moluscos triploides en ambientes ricos en alimento.

Crecimiento. En el grupo de los Ostréidos (Crassostrea gigas, Crassostrea virginica, Ostrea edulis, Saccostrea commercialis), especies para las cuales existe la mayor cantidad de estudios comparativos de crecimiento en triploides versus diploides, se ha observado que los triploides presentan incrementos porcentuales en biomasa húmeda de 29 al 80\% sobre los diploides (Barber y Mann, 1991; Akashige y Fushimi, 1991; Matthiessen y Davis, 1992; Hawkins et al., 1994; Nell et al., 1994; Hand et al., 1998; Landau y Guo, 1999; Ascencio-Michel, 2008; Normand et al., 2009; Callam et al., 2016). El segundo lugar en estudios de este tipo lo ocupa el grupo de los Pectínidos (Argopecten irradians, Argopecten ventricosus, Chlamys nobilis, Chlamys farreri, Nodipecten subnodosus, Patinopecten yessoensis), en donde se ha observado que los triploides presentan un incremento porcentual respecto a los diploides de 32 a $81 \%$ en biomasa húmeda, y un incremento de 44 al 167\% en músculo aductor (Tabarini, 1984; Komaru y Wada, 1989; Ruíz-Verdugo et al., 2000; Yang et al., 2000; MaldonadoAmparo et al., 2004; Meng et al., 2012). En el grupo de los Pteridos, en la especie Pinctada martensii, los triploides presentaron un incremento porcentual de 27 a 58\% sobre los diploides en biomasa húmeda (Jiang et al., 1991). En el grupo de los Mactrídos, la almeja Mulinia lateralis triploide presenta un incremento en biomasa húmeda de $72 \%$ sobre la almeja diploide (Guo y Allen, 1994a). (Tabla 2).

Otras causas de un mejor crecimiento o talla en los triploides. Entre las posibles causas de un mejor crecimiento en triploides con respecto a los diploides están la ya mencionada esterilidad parcial o total, y la 
consecuente canalización alterna de energía que sería utilizada para madurar hacia el crecimiento somático. Esta causa se planteó primero en base a observaciones en Crassostrea gigas (Allen y Downing, 1986). Dos hipótesis alternas han sido propuestas posteriormente para explicar el mayor crecimiento en triploides. La primera propone la ocurrencia de un gigantismo poliploide, causado por el incremento en tamaño celular debido a un incremento en volumen celular sin reducción del número de células en organismos poliploides (Guo y Allen, 1994a). Esta hipótesis, plantea que el gigantismo poliploide puede presentarse solo en algunos órganos como el músculo aductor, y su expresión puede ser afectada por factores ambientales. La segunda hipótesis alterna señala que la ventaja en crecimiento de los triploides es debido a que estos tienen mayor heterocigosidad (alta variación alélica), debido a la probabilidad de llevar tres diferentes alelos en el mismo locus comparado con los diploides. Esta hipótesis fue planteada en observaciones de Crassostrea virginica donde se produjeron triploides de dos formas; inhibiendo el primer cuerpo polar ó el segundo para comparar su crecimiento por tres años. Stanley et al. (1984) observaron que los triploides derivados de la meiosis I presentaron mejor crecimiento debido a que durante la evaluación presentaron mayor heterocigosidad que los producidos inhibiendo la meiosis II. Por otro lado, Jeung et al., (2016), cuantificaron el esfuerzo reproductivo del ostión del Pacífico triploide contrastado con los diploides durante la fase de desove.

Problemática actual en la producción de triploides. Regularmente y como ya se ha mencionado, los triploides son producidos en su mayor parte por la inhibición del segundo cuerpo polar utilizando citocalasina-B (Allen et al., 1989). Sin embargo es importante señalar que existen varias desventajas en la producción de triploides químicos. La primera es que la CB es considerada un químico tóxico y carcinogénico, por lo que requiere un manejo cuidadoso. Aun más, el uso de este químico en algunos laboratorios de moluscos esta bajo revisión por la FDA en EUA (Pitts, 1994, citado por Guo y Allen, 1994b). La segunda desventaja es que la eficiencia del método de inducción actual, aunque ha mejorado, pocas veces resulta en el 100\% de triploidía, y depende de algunos factores ambientales por lo que la incertidumbre en la eficiencia de inducción es una preocupación continua para el manejo de laboratorios (Allen et al., 1989). La tercera desventaja es que el bloqueo del segundo cuerpo polar con CB (o algunos otros agentes) afecta negativamente la supervivencia y crecimiento inicial de los triploides inducidos químicamente (Chew, 1994; Guo y Allen, 1994a; Guo et al., 1996). Finalmente la cuarta desventaja es que se ha observado que en algunas especies los triploides producidos con métodos químicos presentan una reversión cromosómica en tallas grandes (Guo et al., 1996), lo cual ha 
resultado en la incapacidad de introducir triploides a zonas donde no son nativos.

Todos estos problemas podrían ser evitados potencialmente si los triploides fueran producidos biológicamente, esto es, por apareamiento de tetraploides y diploides.

Historia-origen de primeros estudios de tetraploidía en moluscos (Tetraploidía en Moluscos). El desarrollo de organismos tetraploides en moluscos ha sido un proceso difícil, siendo la descripción más acertada de este proceso la realizada por Chew (1994), quien señala que en 1988 el Dr. $\mathrm{Xi}$ Ming Guo fue el primer interesado en el desarrollo de moluscos tetraploides (ostras) durante sus estudios de postgrado con el Dr. Hershberger y el Dr. Chew en la escuela de pesquerías de la Universidad de Washington (UW). En esa época, después de múltiples intentos de producir tetraploides por medio de la inhibición de la primera división celular, y con logros únicamente hasta estadio de larva “D”, la mayoría de la gente en el campo pensó que las ostras tetraploides eran inviables. Guo y sus colegas de la Universidad de Washington iniciaron evaluando una variedad de métodos de inducción a la tetraploidía, incluyendo inhibición de la mitosis I, bloqueo del cuerpo polar I, ginogénesis y fusión celular (Guo et al, 1992a; 1992b; Guo et al,. 1993; Guo et al., 1994). Todos los anteriores trabajos produjeron niveles significantes de embriones tetraploides (19-95\%), pero ninguno de esos embriones sobrevivió a la talla adulta, y la mayoría raramente sobrevivió a larva “D”. Durante esos experimentos se observó que los embriones tempranos de tetraploides eran más grandes que los normales diploides, lo cual no es sorprendente, ya que las células tetraploides contienen el doble de la carga cromosómica de los diploides, por lo que se infirió que necesitan mayor citoplasma para ser funcionales. Esto llevó a Guo y sus colegas a conseguir ovocitos grandes de ostión producidos por diploides, pero se dieron cuenta que estos ovocitos presentan muy poca variación en talla. Durante estos estudios otros investigadores publicaron resultados que abrieron el camino para el futuro desarrollo de tetraploides. Stephens y Downing (1989), publicaron que los ovocitos de las ostras triploides eran significativamente más grandes que los de las diploides normales, de lo cual surgió la idea de utilizar ovocitos de organismos triploides para la obtención de tetraploides. Paralelamente al analizar la fecundidad de organismos triploides de ostión, Guo encontró que las hembras triploides pueden producir un número significante de ovocitos, $\mathrm{y}$ que éstos presentaban divisiones meióticas posteriormente a la fertilización. Estos dos resultados, mayor tamaño en ovocitos de triploides que los de diploides, y la viabilidad meiótica de los ovocitos de triploides, permitieron que a finales de 1990, Guo planteara la hipótesis que establecía que los tetraploides podrían teóricamente ser producidos bloqueando el primer 
cuerpo polar en ovocitos de triploides (fecundados con esperma haploide). En el verano de 1992, Guo fue invitado por el Dr. Allen a la Universidad de Rutgers, Haskin Shellfish Research Laboratory para investigar durante su posdoctorado la genética y el potencial reproductivo de ostras triploides. Guo y Allen (1994c) encontraron que las hembras triploides pueden producir de 1 a 4 millones de ovocitos, y observaron que aunque a una frecuencia baja, los tetraploides podían ser producidos espontáneamente por apareamientos naturales de hembras triploides y machos diploides. En 1993, Guo y Allen probaron la hipótesis, produciendo tetraploides al bloquear con citocalasina B el primer cuerpo polar en ovocitos de triploides fertilizados con espermas haploides. Tres meses posfertilización estos investigadores obtuvieron 2,500 ostiones juveniles, de los cuales 67\% fueron tetraploides, marcando estos estudios el nacimiento de los primeros moluscos tetraploides (Guo y Allen, 1994b).

Métodos de inducción a la tetraploidía. Antes del desarrollo de Guo y Allen en 1994b, tres métodos de inducción a la tetraploidía fueron evaluados en diferentes especies. El primer método involucra la producción incidental de tetraploides, inhibiendo la extrusión del primer cuerpo polar en ovocitos derivados de reproductores diploides. En repetidos estudios se ha demostrado que los embriones producidos por este método (con una excepción) no alcanzan a sobrevivir a semilla o talla adulta (Stephens y Downing, 1988; Dufy y Diter, 1990; Gendreau y Grizel, 1990; Guo et al., 1992a; 1992b; Scarpa et al., 1993; Allen et al., 1994; Cai y Beaumont, 1996; Yang et al., 2000; Zhang et al., 2000). De acuerdo a Allen et al. (1994), existe una especie Ruditapes philippinarum donde se han producido en forma incidental tetraploides que sobrevivieron hasta la talla adulta. El segundo método consiste en la inhibición de la primera división celular (mitosis I) en ovocitos derivados de reproductores diploides, fecundados con espermas haploides, el cual tampoco ha mostrado resultados satisfactorios (Dufy y Diter, 1990; Guo, 1991, citado por Guo y Allen, 1994b; Guo et al., 1994; Yang et al, 1997). El tercer método es el ya descrito como el que ha demostrado su factibilidad en la producción de tetraploides, esto es, utilizando ovocitos producidos por organismos triploides, los cuales son fecundados con espermas de organismos diploides, e inhibiendo la extrusión primer cuerpo polar pero permitiendo la liberación del segundo cuerpo polar (Guo y Allen, 1994b).Tetraploides viables a talla adulta han sido logrados en dos especies, Crassostrea gigas (Guo y Allen 1994b) y Pinctada martensii (He et al., 2000). Estos últimos autores demostraron que la producción de tetraploides en Pinctada martensii también podía ser lograda utilizando el método de Guo y Allen (1994b). Eudeline y Allen (2000), optimizaron la inducción a la tetraploidía en el ostión del Pacífico Crassostrea gigas, usando como indicador natural el primer cuerpo polar. Así mismo, se 
realizaron evaluaciones de inducción a la tetraploidía en almeja Catarina (Argopecten ventricosus) con tres modalidades con el fin de producir tetraploides viables. En la primera, se indujo a la tetraploidía al inhibir la extrusión del primer cuerpo polar en huevos producidos por diploides y fertilizados con esperma haploide, lo cual produjo un porcentaje reducido de larva D tetraploide, pero no resultó en la presencia de semilla o adultos tetraploides. En la segunda, se inhibió la primera división celular del cigoto, resultando principalmente en larva anormal. Cuando la inducción a la tetraploidía se realizó inhibiendo el primer cuerpo polar en huevos de triploide s fertilizados con esperma haploide se obtuvo un alto porcentaje de larva D tetraploide en una de las inducciones. De las progenies tetraploides seis sobrevivieron a talla adulta. En base a lo anterior, para esta especie en particular, la producción de tetraploides deberá centrarse en esta última modalidad (Maldonado-Amparo et al., 2003). Yang y Guo (2004), evaluaron en Mulinia lateralis (Say 1822), el efecto de la duración de la Citocalasina B en la inducción a la tetraploidía al inhibir la meiosis.

Características generales del tetraploide. La característica de más importancia que debe tener un tetraploide es el ser fértil, ya que esto permitirá el perpetuar su descendencia y producir los denominados "triploides biológicos". Guo et al. (1996) produjeron en el ostión Crassostrea gigas triploides biológicos a partir de los tetraploides logrados, evaluando comparativamente estos con triploides producidos con CB. Entre los resultados más significantes estuvo el hecho de que se logró eliminar en esta especie uno de los problemas asociados con los triploides inducidos químicamente, ya que los triploides biológicos a 8 meses de edad eran 100\% triploides, mientras que en los triploides producidos con CB hubo reversión cromosómica, habiendo encontrado solo 46\% triploides y 2\% mosaicos (diploide-triploide). Posteriormente Guo y Allen (1997), evaluaron la gametogénesis, proporción de sexo y el proceso meiótico en tetraploides, triploides y diploides en Crassostrea gigas, encontrando que los tetraploides alcanzaron la madurez sexual en un año, presentando una relación de sexos aproximada de 1:1. En contraste con una frecuencia alta de hermafroditas en los triploides, en Crassostrea gigas tetraploide hubo aproximadamente el mismo nivel de hermafroditas que en los diploides. La fecundidad de los tetraploides fue comparable a la de los diploides, difiriendo mucho de una reducida fecundidad de los triploides. En relación al proceso meiótico en triploides, tres cromosomas homólogos sinapsan como trivalentes y en la metafase de la meiosis I el cromosoma extra segrega en forma aleatoria produciendo gametos aneuploides. Sin embargo, en tetraploides los cuatro cromosomas sinapsan de forma cuadrivalente y los dos cromosomas extras cosegregan en la meiosis I para producir gametos balanceados diploides. 
Bases del conocimiento celular asociado con la inducción a la poliploidía (Mitosis).Al igual que la mayoría de las especies animales los moluscos tienen dos juegos de cromosomas (cada juego siendo la contribución de cada progenitor) y son referidos como diploides. El crecimiento de un organismo diploide se lleva a cabo por multiplicación o división celular, implicado únicamente el proceso mitótico (Fig. 1). Una célula diploide experimenta un periodo de crecimiento e incremento de la masa celular, denominada $\mathrm{G}_{1}$. En el caso de una célula que requiere 24 horas para el ciclo completo, las primeras 10 horas podrían corresponder al periodo G1. En este periodo la célula además de dedicarse a crecer se prepara químicamente para la síntesis de ADN. Durante la fase de síntesis (S), cuya duración es de alrededor de 9 horas, el material genético (ADN de todos los cromosomas) se duplica. Después de completarse la duplicación del ADN, la célula entra en una segunda fase de crecimiento denominada $\mathrm{G}_{2}$. Esta etapa requiere de por lo menos 4 horas y continúa hasta la cuarta fase, correspondiente al inicio de la mitosis, la cual se completa en sólo una hora (Gardner et al., 1998).

La mitosis, que en un sentido estricto se refiere a la división del núcleo en dos núcleos hijos idénticos seguida por una división celular, comprende cuatro fases sin considerar la interfase, que es el lapso que transcurre entre dos divisiones celulares $\left(\mathrm{G}_{1}, \mathrm{~S}, \mathrm{G}_{2}\right)$. Estas fases son profase, metafase, anafase y telofase (Fig. 2). Una vez que los cromosomas se duplican durante la interfase $\left(G_{1}, S, G_{2}\right)$ los centríolos (dos pequeños cuerpos cilíndricos, los cuales desempeñan un papel prominente durante la división celular; se separan, emigran a los polos opuestos de la célula y organizan el huso entre ellos) se dividen e inician su separación hacia los polos opuestos de la célula. Durante la profase los cromosomas se compactan y pueden observarse en un microscopio óptico que son dobles en toda su extensión, excepto en un pequeño punto en el que están unidos, llamado centrómero. Cada una de las dos partes idénticas (replicadas) de un cromosoma recibe el nombre de cromátida.

Los centríolos se desplazan a los lados opuestos del núcleo y el citoplasma que los rodea se modifica formando fibras proteicas delgadas que darán lugar al huso acromático. Al conjunto formado por el centríolo y los filamentos de la proteína tubulina se les conoce como aster. Al final de la profase, tanto la membrana nuclear como los nucleolos se desintegran y desaparecen en el citoplasma. Los cromosomas replicados se desplazan hacia el ecuador, y en ese momento la mitosis entra en etapa de metafase. En esta fase los cromosomas se unen a las fibras del huso acromático por medio de su centrómero. Durante laanafase, los centrómeros se separan y tan pronto como esto sucede las cromátidas hermanas de cada cromosoma se repelen mutuamente, iniciando un desplazamiento hacia polos opuestos, 
posiblemente debido al acortamiento de las fibras del huso que jalan a las cromátidas. La anafase termina cuando las cromátidas llegan a los polos (cada cromátida es ahora un cromosoma completo). Después de llegar a los polos se alargan o descompactan, estado en el que estarán durante la interfase. Durante la telofase las fibras del huso acromático y los aster desaparecen, se forman dos núcleos con una membrana alrededor de cada uno, y la mitosis concluye. Finalmente el citoplasma se divide mediante una constricción que converge de las dos partes separando a las dos células hijas, ambas entrando a una nueva interfase (Fig. 2), (Gardner et al., 1998).

Meiosis.La meiosis, a diferencia de la mitosis, es un proceso en el cual adicionalmente a la replicación cromosómica, existen dos reducciones cromosómicas con el fin de obtener un producto haploide. Otra característica que distingue a la meiosis de la mitosis es el entrecruzamiento o recombinación entre cromosomas homólogos (proceso por el cual los cromosomas homólogos intercambian segmentos formando nuevas combinaciones de alelos). La meiosis comienza en un núcleo diploide de células germinales, en el cual cada cromosoma contiene una cantidad de ADN replicado. Después de dos divisiones celulares (meiosis I y meiosis II) y en ausencia de una nueva replicación de ADN, se obtienen cuatro núcleos haploides en los cuales cada juego cromosómico contiene la mitad de la cantidad de ADN que había antes del inicio de la meiosis (Fig. 3), (Gardner et al., 1998).

Primera división meiótica (MI). La profase de la meiosis I (primera división) se subdivide en subetapas. La profase es el intervalo más complejo, prolongado y genéticamente más significativo, debido al entrecruzamiento o recombinación entre cromosomas homólogos que permite generar nueva variabilidad genética en los gametos (Fig. 3.1). La subdivisiones de la profase I son: leptoteno ("filamento delgado"), cigoteno ("filamento pareado"), paquiteno (“filamento grueso"), diploteno (“filamento doble”) y diacinesis ("dividido a través"). En el inicio de la profase I, en leptoteno, los filamentos de cromatina se ven mezclados, y resulta casi imposible seguir un filamento por más de una micra de su longitud sin perderlo en un laberinto de otros filamentos Fig. 3.1A). La continuación de la profase $I$ es el cigoteno, caracterizado por ser el estado donde ocurre el apareamiento de los cromosomas homólogos, a travéz del proceso de asociación denominado sinápsis. La sinápsis no parece comenzar en un sitio particular del cromosoma, ni tampoco ciertos cromosomas se juntan antes que otros. A medida que ocurre la sinápsis, en el espacio que queda entre los cromosomas apareados se organiza una estructura compleja, denominado complejo sinaptonémico, la cual se encuentra exclusivamente en las células meióticas durante la profase I temprana. El complejo sinaptonémico se caracteriza por apareamiento punto por punto de cromosomas homólogos dando lugar 
formaciones cromosómicas que se conocen como bivalentes (Fig. 3.1B y F). Cuando termina el apareamiento y el complejo sinaptonémico esta completamente desarrollado, el cigoteno termina e inicia el paquiteno, el cual se caracteriza por presentar los cromosomas homólogos completamente apareados. Los cromosomas se han condensado mucho más, aunque todavía no se muestra visualmente su condición replicada. Un par de cromosomas es llamado bivalente, y al igual que para la mitosis, cada cromosoma de este par está constituido por dos mitades replicadas denominadas cromátidas hermanas. A medida que el paquiteno avanza los bivalentes se condensan más, pero siguen siendo pares en estrecha sinapsis. El intercambio físico entre segmentos de cromosomas homólogos, proceso denominado entrecruzamiento (“crossing over”), ocurre durante el paquiteno (Fig. 3.1C). En el diploteno comienza la separación de los cromosomas sinapsados a lo largo de diversos sectores, y los homólogos se mantienen asociados sólo en ciertos lugares denominados quiasmas, que son los sitios en donde previamente ocurrió entrecruzamiento entre cromátida diferentes homologas (Fig. 3.1D). La diacinesis se caracteriza por la máxima contracción de los cromosomas. Los cromosomas bivalentes se observan cortos y gruesos, los quiasmas son evidentes, y los bivalentes individuales están relativamente bien separados en el núcleo. A medida que la diacinesis se aproxima a su término, el núcleo y la envoltura nuclear desaparecen y los microtúbulos del huso se hacen visibles en el área cromosómica (Fig. 3.1E). En prometafase los cromosomas se mueven de manera errática, pero eventualmente ocurre la conjunción de cromosomas homólogos en el punto medio del huso. Al final de esta fase preliminar los bivalentes homólogos se encuentran alineados conjuntamente a lo largo del plano ecuatorial del huso, iniciando así la metafase I. En esta fase los brazos de los cromosomas bivalentes se encuentran en el ecuador del huso y los centrómeros de los cromosomas bivalentes homólogos se encuentran pareados pero tan separados entre sí como físicamente es posible. Esta situación es opuesta a la del alineamiento de los cromosomas durante una metafase mitótica, en la que los centrómeros se ubican en el punto medio del huso de cada uno de los cromosomas replicados (sin apareamiento de homólogos) y los brazos de los cromosomas se ondean en todas direcciones de ambos lados de esta zona. (Gardner et al., 1998).

Cada cromosoma del bivalente está compuesto por dos cromátidas y los centrómeros de cada par de cromátidas hermanas permanecen estrechamente asociadas al mismo lado del punto medio del huso, esto es, enfrentan el mismo polo, mientras que las cromátidas hermanas del cromosoma homólogo enfrentan el polo opuesto. Cuando laanafase I inicia, cada cromosoma completo (replicado y recombinado) se separa de su homólogo dirigiéndose hacia polos opuestos. Cada cromosoma es conocido 
como diada (describe su carácter doble e indica que esta compuesto por dos cromátidas). Cada diada posee la cantidad $2 \mathrm{X}$ de $\mathrm{ADN}$, de tal forma que la reducción a la haploidía aun no se alcanza. En telofase I, si es que esta etapa existe en una secuencia meiótica, la reorganización de los cromosomas ocurre. Los cromosomas sé descondensan y alcanzan longitudes mayores, los núcleos y envolturas nucleares reaparecen haciéndose así evidentes los núcleos hijos, ocurriendo posteriormente la citocinesis (Fig. 3) (Gardner et al., 1998).

Segunda división meiótica (MII).De la primera a la segunda división meiótica no existe interfase ni profase, iniciandose esta división en la metafase. Cada cromosoma compuesto de dos cromátidas hermanas pero recombinadas se desplaza independientemente del homologo hasta el ecuador en la metafase II. En laanafase II, las cromátidas de cada cromosoma duplicado se separan, y migran hacia los polos opuestos del huso, cada uno de ellos siendo un cromosoma. En la telofase II cada cromosoma se descompacta al acercarse la interfase. Al terminar la meiosis II existen cuatro células sexuales haploides (Gardner et al., 1998).

Diferencias entre los gametos sexuales formados. Mientras que cada célula germinal masculina produce cuatro gametos haploides funcionales después de haber completado la meiosis I y II, la célula germinal femenina se divide en forma desigual, de tal forma que en la primera división meiótica una de las dos células contiene casi todo el citoplasma y un juego de cromosomas replicados, y la otra no contiene más que el otro juego de cromosomas replicados. Esta última célula es denominada como primer cuerpo polares cual permanece adherido a la célula con el mayor citoplasma. La meiosis II sigue un patrón similar en cuanto a la distribución de citoplasma entre las dos células formados en esta división, pero ahora al concluir esta cada una contiene un juego de cromosomas haploide. A la célula que contiene solo cromosomas y no citoplasma se le denomina segundo cuerpo polar. Al final de la meiosis se produce un ovocito con dos cuerpos polares; el segundo cuerpo polar (haploide) adherido al ovocito, e intermedio al primer cuerpo polar (diploide) posicionado distalmente (Beaumont y Fairbrother, 1991) (Fig. 3).

La inducción a la triploidía y su asociación con eventos meióticos. En los moluscos los ovocitos son expulsados al ambiente para la fecundación externa. Su desarrollo meiótico esta detenido en la profase de la meiósis I. El proceso meiótico se reinicia una vez que ocurre la fertilización por el esperma, (Longo, 1983). Siendo esta característica la que permite la inducción artificial a la poliploidía en la mayor parte de los moluscos.

Inhibición de la Meiosis I. Cuando se inhibe el primer cuerpo polar (meiosis I) para producir triploides, los cromosomas replicados de cada homólogo que formarían el primer cuerpo polar son retenidos dentro del 
ovocito. Posteriormente se permite que la meiosis II ocurra la cual diferirá de un ovocito en el cual no se inhibió el primer cuerpo polar en que el doble de cromosomas tendrá que alinearse en forma individual en la placa metafásica para la segunda división ecuacional, y el segundo cuerpo polar contendría dos juegos de cromosomas al igual que el ovocito, esto es, contendrían $2 \mathrm{X}$ de ADN, y serian diploides. Al unirse estos cromosomas con el juego de cromosomas proveniente del esperma se forma un cigoto triploide (Fig. 4) (Beaumont y Fairbrother, 1991). Si la segregación cromosómica durante la meiosis II no es balanceada, otro tipo de ploidías pueden ocurrir. Por ejemplo, tetraploides pueden ocurrir cuando en el segundo cuerpo polar solamente sé extruye un juego cromosómico, reteniéndose en el ovocito tres juegos completos derivados de un homologo replicado, y uno del otro homologo replicado. Al ocurrir la singamia ( fusión del pronúcleo femenino y masculino) de pronúcleos femenino y masculino, el cigoto resultante será tetraploide. Es importante mencionar que en estudios donde se ha inhibido "teóricamente" solo la MI para producir triploides, se ha observado la ocurrencia en estadios larvarios de tetraploides y pentaploides. Estos últimos son resultado de inhibir la MI y MII lo que resulta en pronúcleos femeninos tetraploides, los cuales al unirse con el pronúcleo masculino haploide darán como resultado un cigoto pentaploide (Cooper y Guo, 1989). La ocurrencia de tetraploides como resultado de la inhibición de la primera división durante la meiosis puede ser explicada como resultado del alineamiento de homólogos bivalentes en la metafase I con una segregación posterior de 3:1.

Inhibición de la Meiosis II. El proceso más común por el mayor éxito logrado para la producción de triploides en moluscos es a través de la inhibición de la formación del segundo cuerpo polar (MII), habiendo permitido formar normalmente el primer cuerpo polar. En este método, en el cual la formación del segundo cuerpo polar es suprimida, se retiene de esta forma a ambas cromátidas hermanas de cromosomas homólogos para formar un pronúcleo femenino diploide, el cual después de unirse con el juego de cromosomas aportado por el esperma forma un cigoto triploide (Fig. 5) (Beaumont y Fairbrother, 1991).

La inducción a la tetraploidía y su asociación con eventos mitóticos y meióticos. La inducción a la tetraploidía ha utilizado algunos métodos: Inhibición de la mitosis o la primera división celular del cigoto. Teóricamente, si el tratamiento de inhibición con CB es administrado durante la primera división, los cromosomas replicados que normalmente se dividirían para formar dos células “hijas”, serían retenidos en una sola célula resultando en un cigoto con cuatro juegos de cromosomas. Subsecuentemente las divisiones mitóticas producirían sólo células con cuatro juegos de cromosomas, conformando un organismo tetraploides (Fig. 6) (Beaumont y Fairbrother, 1991).El segundo método es a través de la 
inhibición del primer cuerpo polar, en donde como ya se explico anteriormente una segregación de 3:1 durante la meiosis II permitiría lograr la producción de tetraploides (Fig. 7).

Inhibición del primer cuerpo polar en huevos de triploides: características de la meiosis en triploides.Al inhibir la meiosis I, o la primera reducción cromosómica en un ovocito de un triploide, se reduce la probabilidad de producir gametos no balanceados. Los gametos no balanceados ocurren debido a que para cada cromosoma existen tres copias en el triploide, y durante la metafase I solamente se podrán parear dos de ellas. La tercera copia de cada uno de los cromosomas podrá migrar aleatoriamente hacia cualquiera de los dos polos durante la anafase I, lo cual resultará en que en algunos casos la tercera copia de un cromosoma migre hacia el cuerpo polar, pero para otros cromosomas la tercera copia puede que sea retenida. Al inhibir la primera reducción cromosómica o meiosis I lo que se busca es que existan cromosomas en pares, de tal manera que durante la segunda reducción cromosómica (metafase II) cada cromosoma se alinea en el ecuador de la célula, lográndose así la producción de gametos balanceados, que se caracterizarán por contener tres juegos completos de cromosomas. Cuando la contribución cromosómica del esperma es integrada, el cigoto producido es tetraploide, en el cual tres juegos son aportados por la hembra, y uno por el macho (Fig. 8).

\section{Conclusión}

Contrario a lo que acurre a nivel internacional en cuanto a la generación de organismos poliploides para ser utilizados en la producción comercial de Moluscos, en México existen pocos avances. Por lo que es importante establecer las bases biotecnológicas para la inducción a la poliploidía en las especies endémicas potenciales de cultivo.Es evidente las ventajas en crecimiento de los organismos triploides para la acuacultura respecto a los diploides en las diversas especies reportadas, debido a la esterilidad, al no gastar energía en la formación de gametos alcanzando mayor talla. Sin embargo, es importante considerarla problemática que existe para producir triploides mediante el uso de diferentes inductores químicos, es esencial realizar mayores esfuerzos dirigidos a la producción de tetraploides en moluscos usando la metodología de Guo y Allen (1994) para producir triploides biológicos. Para lo cual se requerirían perpetuar líneas de de tetraploides con el fin de mantener un stock de reproductores que al ser apareados con diploides permita la producción de triploides biológicos.

\section{Agradecimientos}

Esta investigación fue soportada por el proyecto de CONACYT 28256-B de la Dra. A.M. Ibarra.El primer autor agradece la Dirección 
General de Educación en Ciencia y Tecnología del Mar de la SEMS de la SEP, por el apoyo para realizar el Posdoctorado dentro del Programa del Periodo Sabático (Agosto 2016-Agosto 2017) en el Programa de Posgrado en Ciencias Marinas y Costeras de la UABCS.

\section{References:}

1. Akashige, S., Fushimi, T., 1991. Growth, survival and glycogen content of triploid pacific oyster Crassostrea gigas in the waters of Hiroshima, Japan. Nippon Suisan Gakkaishi 56: 1063-1071.

2. Allen, S. K. Jr., 1987a. Gametogenesis in three species of triploid shellfish: Mya arenaria, Crassostrea gigas and Crassostrea virginica. Proc. World Symposium on Selection Hybridation and Genetic Engineering in Aquaculture of fish and shellfish for consumption and stocking. Bordeaux, 27-30 may, Vol. II, Berlin, pp. 207-217.

3. Allen, S. K. Jr., 1987b. Genetic manipulations - Critical Review of methods and performances. proc. World Symposium on selection Hybridization and Genetic Engineering in aquaculture of fish and shellfish for consumption and stoking. Bordeux, 27-30 May. Voll II, Berling. 127-143 pp.

4. Allen, S. K. Jr. 1988. Triploid oysters ensure year-round supply. Oceanus, 58-63.

5. Allen, S. K. Jr., Bushek, D., 1992. Large-scale production of triploid oysters, Crassostrea virginica (Gmelin), using "stripped” gametes. Aquaculture, 103: 241-251.

6. Allen, S.K. Jr., Downing, S.L., 1986 Performance of triploid pacific oyster, Crassostrea gigas (Thunberg). I Survival, growth, glycogen conten and sexual maturation in yearlings. J. Exp. Mar. Biol.. Ecol., 102: 197-208.

7. Allen, S.K. Jr., Downing, S.L., 1991. Performance of triploid Pacific Oysters Crassostrea gigas, gametogenesis. J. Fish. Aquat. Sci. (47): 1213-1222.

8. Allen, S.K. Jr., Downing, S.L., Chew, K.K., 1989. Hatchery Manual for Producing Triploid Oysters. Washington Sea Grant Program, University of Washington Press. pp. 27.

9. Allen, S.K.Jr., Gagnon, P.S., Hidu, H., 1982. Induced triploidy in the soft-shell clam. Cytogenetic and allozymic confirmation. The journal of Heredity 73: 412-428.

10. Allen, S. K. Jr., Hidu, H., Stanley, J.G., 1986. Abnormal gametogenesis and sex ratio in triploid soft-shell clams (Mya arenaria). Biol. Bull. 170: 198-210. 
11. Allen, S. K. Jr., Shpigel, M., Utting, S., Spencer, B., 1994. Incidental production of tetraploid Manila clams Tapes Philippinarum (Adams and Reeve) Aquaculture, 128: 13-19.

12. Ascencio-Michel, R. 2008. Análisis comparativo del crecimiento y supervivencia del ostión Crassostrea gigas diploide y triploide en diferentes medios ambientales del Noroeste de Mexicano. Tesis de licenciatura. UABCS. 80 p.

13. Barber B.J., Blake N.J., 1991. Reproductive physiology. In: Shumway, S.E. (Ed).Scallops: Biology, Ecology an Aquaculture. Elsevier, Amsterdam, pp. 377-428.

14. Barber, B.J., Mann, R., 1991. Sterile triploid Crassostrea virginica (Gmelin, 1791) growth faster than diplid but are equally susceptible to Perkinsus marinus. Journal of Shellfish Research 10(2): 445-450.

15. Barber, B.J., Mann, R., Allen, S.K.Jr., 1992. Optimization of triploid induction for the oyster Crassostrea virginica (Gmelin). Aquaculture 106: 21-26.

16. Baron, J., Diter, A., Bodoy, A., 1989. Triploidy Induction in the Black Scallop Chlamys varia L.) and its effect on larval growth and survival. Aquaculture, 77:103-111.

17. Beaumont, A.R., 1986. Genetic aspects of hatchery rearing of the Scallop Pecten maximus (L) Aquaculture, 57:99-110.

18. Beaumont, A. R., Fairbrother, J.E., 1991. Ploidy manipulation in mollusca shellfish: a review. J. Shellfish Res., 10(1): 1-18.

19. Beaumont, A. R., Fairbrother, J.E., Hoare, K., 1995. Multilocus heterozysity ana size: A test of hypotheses using triploid Mytilus edulis. Heredity 75(3): 256-266.

20. Beaumont, A.R., Kelly, K.S., 1989. Production and growth of triploid Mytilus edulis larvae. J. Exp. Mar. Biol. Ecol. 132: 69-84.

21. Brake, J., Dvaidson, J., Davis, J., 2002. Observations on growth, gametogenesis and sex ratio of triploid and diploid Mytilus edulis. Journal of Shellfish Research 21(1): 392.

22. Cai, G., Beaumont, R., 1996. Tetraploid induction in the mussel Mytilus edulis by application of 6-dimethylaminopurine (6-DMAP) during early development. Tropic Oceanology/Redai Haiyang, 15: 26-30.

23. Callam, B.R., Allen J.S.A. y Frank-lawale, A. 2016. Genetic and environmental influence on triploid Crassostrea virgínica grown in Chesapeake Bay: Growth. Aquaculture 452: 97-106.

24. Canello, O. F., Paredes, O.L., Toro, Y. J., 1992. Inducción a la triploidía en el ostión del norte Argopecten purpuratus, por medio de shock térmico de calor. Inv. Pesq. (Chile) 37: 5-11. 
25. Chew, K. K., 1994. Tetraploid Pacific Oyster offer promise to future production of triploid. Aquaculture magazine november/december, pp. 69-74.

26. Cooper, J., Guo, X., 1989. Poliploid Pacific oysters produced by inhiting polar body I and II with cytochalasin B. Journal of Shellfish Res., 8412

27. Cox, E.S., Smith, M.S.R., Nell, J. A., Maguire, G.B., 1996. Studies on triploid oysters in Australia. VI. Gonad development in diploid and triploid Sydney rock oysters Saccostrea commercialis (Iredale and Roughley). Aquaculture, 197: 101-120.

28. Desrosiers, R.R., Gerard, A., Peignon, J.M., Naciri, Y., Dufresne, L., Morasse, J., Ledu, C., Phelipot, P., Guerrier, P., Dube, F., 1993. A novel method to produce triploid embryos in bivalve mollusks by the use of 6-dimethylaminupurine. J. Exp. Mar. Biol. Ecol. 170: 29-43.

29. Downing, S.L., Allen, S.K. Jr., 1987. Induced Triploidy in the Pacific Oyster, Crassostrea gigas: optimum treatments with Cytochalasin B Depend on Temperature. Aquaculture, 61: 1-15.

30. Dufy, C., Diter, A., 1990. Poliploidy in the Manila Clam Ruditapes philippinarum. II. Chemical induction of tetraploid embryos. Aquat. Living Resour. 3: 107-112.

31. Dunstan, G. Elliot, N., Appleyard, S., Holmes, B., Conod, N., Grubert, M., Cozens, N., 2007. Culture of triploid greenlip abalone (Haliotis laevigata Donovan) to market size: comercial implications. Aquaculture 271 (1-4): 130-141.

32. Eudeline, B., AllenJ. S.K., 2000. Optimization of tetraploid induction in pacific oysters Crassostrea gigas using first polar body as a natural indicator. Aquaculture 187 (1-2): 73-84.

33. Eversole, A.G., Kempton, C.J., Hadley, W. H., Buzzi, W.R., 1996. Comparison of growth, survival, and reproductive success of diploid and triploid Mercenaria mercenaria. Journal of Shellfish Research 15(3): 689-694.

34. FAO. 2003. Review of the state of word aquaculture. FAO Fisheries Circular No. 886, Rev. 2, Rome, FAO 2003. 95 p.

35. Felix-Pico, E.F., Villalejo-Fuerte, M., Pripp-Quezada, A., HolguinQuiñones, O., 1999. Growth and survival of Lyropecten subnodosus (Sowerby, 1835) in suspensed cultured at the National Marine Park of Bahia de Loreto, B.C.S., México. 12 Int. Pectinid Worshop, Bergen (Norway) 5-11, May. Book-abstracts.

36. García, L., Salas, A., Searcy, R., García, F., Lizárraga, S., Cancio, J. L., 1988. Cultivo experimental de mejillón Mytilus edulis en la Bahía de Todos Santos, B.C. Informe Anual, Tomo I. Cap. 2; 68-151 pp. U.A.B.C. México. 
37. Gardner, E.J., Simmons, M.J., Snustad, D.P., 1998. Principios de Genética. Cuarta edición. Noriega editores. México, D.F.

38. Gendreau, S., Grizel, H., 1990. Induced triploidy and tetraploidy in European flat oyster, Ostrea edulis. Aquaculture, 90:229-238.

39. Gerard, a., Naciri, Y., Noiret, C., Ledu, C., Peignon, J.M., Phelipot, P., 1994a. Induced triploidy in the European clam, Ruditapes decussatus (L.) and performance of triploid larvae. Aquaculture and Fisheries Management, 25: 769-779.

40. Gerard, A., Naciri, Y., Peignon, J.M., Ledu, C., Phelipot, P., 1994b. Optimization of triploid induction by the use of 6-DMAP for the oyster (Crassostrea gigas Thunberg). Aquaculture and Fisheries Management 25: 709-919.

41. Gerard, A., Ledu, C., Phelipot, P., 1999. The induction of MI and MII triploids in thepacific oyster Crassostrea gigas with 6-DMAP or CB. Aquaculture 174: 229-242.

42. Gosling, E.M., Nolan, A., 1989. Triploidy induction by thermal shock in the manila clam, Tapes semidecussatus. Aquaculture 78: 223-228.

43. Guo, X., Allen, S.K. Jr., 1994a. Sex Determination and Polyploid Gigantism in the Dwarf Surfclam (Mulinia lateralis Say) Genetics, 138: 1199-1206.

44. Guo, X., Allen, S.K. Jr., 1994b. Viable tetraploids in the pacific Oyster (Crassostrea gigas Thunberg) produced by inhibition polar body 1 in eggs from triploids. Molecular Marine Biology and Biotecnology, 3(1): 42-50.

45. Guo, X., Allen, S.K. Jr., 1994c. Reproductive potential and genetic of triploid Pacific oysters, Crassostrea gigas (Thunberg) Biol. Bull. 187: 309-318.

46. Guo, X., Allen, S.K. Jr., 1997. Sex and meiosis in autotetraploid Pacific oyster, Crassostrea gigas(Thunberg). Genome, 40:397-405.

47. Guo, X., DeBrosse, G.A., Allen, S.K. Jr. 1996. All-triploid Pacific Oysters (Crassostrea gigas Thunberg) produced by mating tetraploid and diploid. Aquaculture, 142: 149-161.

48. Guo, X., Cooper, K., Hershberger, W. K., Chew, K.K., 1992a. Genetic consequences of blocking polar body I with cytochalasin B in fertilized eggs of Pacific oyster, Crassostrea gigas: 1. Ploidy of resultant embryos. Biol. Bull., 183: 381-386

49. Guo, X., Hershberger, W.K., Cooper, K., Chew, K.K., 1992 b. Genetic consequences of blocking polar body I with Cytochalasin B in fertilized eggs of Pacific Oyster, Crassostrea gigas II Segregation of chromosomes, Biol. Bull. (Woods Hole, Mass), 183: 387-393. 
50. Guo, X., Hershberger, W.K. Cooper, K., Chew, K.K., 1993. Artificial gynogenesis with ultraviolet light-irradiated sperm in the Pacific oyster, Crassostrea gigas. I. Induction and survival. Aquaculture 113: 201-214.

51. Guo, X., Hershberger, W.K. Cooper, K., Chew, K.K., 1994. Tetraploid induction with mitosis $\mathrm{l}$, inhibition and cell fusion in the Pacific oyster (Crassostrea gigas Thunberg). J. Shellfish Res., 13: 193-198.

52. Hand, R., Nell., J., Maguire, G.B., 1998. Studies on triploid oysters in Australia: X. Growth and mortality of diploid and triploid sydney rock oysters Saccostrea commercialis (Iredale and Roughley). Journal of Shellfish Research 17(4): 1115-1127.

53. Hawkins, A.J.S., Day, A.J., Gerard, A., Naciri, Y., Ledu, C., Bayne, B.L., Heral, M., 1994. A genetic and metabolic basis for faster growth among triploids by blocking meiosis I but not meiosis II in the larviparus european flat oysters, Ostrea edulis L. J. Exp. Mar. Biol. Ecol. 184: 21-40.

54. He, M., Lin, Y., Shen, Q., Hu, J., Jiang, W., 2000. Production of tetraploid pearl oyster (Pinctada martensii Dunker) by inhibiting the first polar body in eggs from triploid. J. Shellfish Res., 19: 147-151.

55. Ibarra-Humphries A.M., Ramirez-Arce, J.L., 2008. Manual Práctico. Inducción a la triploidía con citocalacina B en ostión Japonés Crassostrea gigas. Centro de Investigaciones Biológicas del Naroeste S.C. (CIBNOR). La Paz, B.C.S., México. 19 p.

56. Jackson, J.E., MacDonald, B.W., James, S., Vercaemer, B., Kenchington, E.L., 2002. Investigations with triploid atalantic sea scallops Placopecten magellanicus. Journal of Shellfish Research 21(1): 433.

57. Jeung, H-D., Keshavmurthy, S., Lim, H-J., Kim, S-K., Choi, K-S., 2016. Quantification of reproductive effort of the triploid Pacific oyster Crassostrea gigas raised in intertidial rack and bag oyster cultura system off the west coast of Korea during spawning season. Aquaculture 464: 374-380.

58. Jiang, W.G., Li, G., Xu, G.Q., Lin, Y.G., Quing, N., 1993. Growth of the induced triploid pearl oyster, Pinctada martensii (D). Aquaculture, 111: 245-253.

59. Jiang, W., Xu, G., Lin, Y., Li, G., 1991. Comparison of growth between triploid and diploid of Pinctad martensii (D.). Tropic Oceanography. 10(3): 1-7. (en chino).

60. Kiyomoto, M., Komaru, K., Scarpa, J., Wada, K.T., Danton, E., Awaji, M., 1996. Abnormal Gametogenesis, Male Dominant Sex 
Ratio, and Sertoli Cell Morphology in Induced triploid Mussel, Mytilus galloprovincialis. Zool. Sci., 13: 393-402.

61. Komaru, A., Wada, K. T:, 1989. Gametogenesis and growth of induced triploid scallops Chlamys nobilis. Nippon Suissan Gakkaishi 55: 447-452.

62. Komaru, A., Wada, K. T:, 1990. gametogenesis of triploid Japanese pearl oyster, Pinctada fucata martensii. In: Goshi, M., Yamashita, , O. (Eds.) Advances in Invertebrate Reproduction \%. Elsevier Science Publisher B.V., Amsterdan, pp. 469-474.

63. Landau, B., Guo, X., 1999. Growth characteristics in triploid pacific oysters - A new dimension. Journal of Shellfish Research 18(1): 270271.

64. Liu, W., Heasman, M., Simpson., 2004. Optimizatión of triploid induction in the blacklip abalone Haliotis rubra (Leach, 1814) using 6-dimetuylaminopurina. Aquaculture Research. 35: 1076-1085.

65. LLohrmam, K.B., Von Brand, E., 2005. Histological study of gonad in triploid scallops Argopecten purpuratus. Journal of Shellfish Research. 24 (2): 369-375.

66. Longo, F.J., 1972. The effects of cytochalasin B on the events of fertilization in the surf clam, Spisula solidissima. J. Exp. Zool. 182: 322-344.

67. Longo, F.J., 1983. Meiotic maturation and fertilization. In: The Mollusca, Vol. 3, Verdonk, N.K., Van Den Biggelar, J.A.M., Tompa, A.S. (eds), Academic Press, New York, pp. 49-83.

68. Maeda-Martínez, A.N., Lombeida, P., Freites, L., Lodeiros, C., Sicard, M.T., 2001. Cultivo de pectínidos en fondo y estanques. En: A.N. Maeda Martínez (Ed) Los moluscos Pectínidos Iberoamericanos: Ciencia y Acuacultura. Editorial LIMUSA, México, D.F.

69. Maldonado-Amparo, R., Ibarra, A.M., Ramirez, J.L., Ävila, S., Vazquez, J.E., Badilla, L.M. 2001. Successful induction of triploidy in Pacific red abalone (Haliotis rufescens). Journal of Shellfish Research. 20 (3): 1071-1075.

70. Maldonado-Amparo, R., Ibarra, A.M. 2002a. Ultrastructural characteristics of spermatogenesis in diploid and triploid catarina scallop (Argopecten ventricosus Sowerby II, 1842). Journal of Shellfish Research 21 (1): 93-101.

71. Maldonado-Amparo, R., Ibarra, A.M. 2002b. Comparative analysis of oocyte type frequencies in diploid and triploid Catarina scallop (Argopecten ventricosus) as indicators of meiotic failure. Journal of Shellfish Research 21 (2): 597-603. 
72. Maldonado-Amparo, R., Ibarra, A.M., Ramirez, J.L. 2003. Induction to tetrapolidy in Catarina scallop, Argopecten ventricosus (Sowerby II, 1842). Ciencias Marinas 29 (2): 229-238.

73. Maldonado-Amparo, R., Ramirez, J.L., Avila, S., Ibarra, A.M. 2004. Triploid lion-paw scallops (Nodipecten subnodosus); grwth, gametogénesis and gametic cell frequencies, when grwn at a high food availability site. Aquaculture 235: 185-205.

74. Matthiessen, G.C., Davis, J. P., 1992. Observations on growth rate and resistance to MSX (Haplosporidium Nelson) among diploid and triploid eastern oysters (Crassostrea virginica Gmelin, 1791) in new England. J. Of Shellfish Res. 11: 449-454.

75. Mazon, S.J.M., 1996. Cultivo de almeja catarina Argopecten ventricosus. En: Estudio del potencial pesquero y acuícola de Baja California Sur. Casas y Valdez, M. y G. Ponce Díaz (eds), México.

76. Melo, E.M.C., Gomes, C.H.A., Silva, F.C., Suhnel, S., Melo, C.M.R., 2015. Chemical and physical methods of triploidy induction in Crassostrea gigas (Thunberg, 1793). Biol. Inst. Pesca, Sao Paulo. 41(4): 889-898.

77. Meng, Q., Bao, Z., Wang, Z., Wang, S., Hu, J., Hu, X., Huang, X., 2012. Growth and reproductive performance of triploid yesso scallops (Patinopecten yessoensis) induced by hypotonic shock. Journal of Shellfish Research 31 (4): 1113-1122.

78. Nell, J.A., Cox, E., Smith, I.R., Maguire, G.B., 1994. Studies on triploid oyster in Australia: I. The farming potential of of triploid Sidney rock oysters Saccostrea commercialis (Iredale and Roughley). Aquaculture 126: 243-255.

79. Nell, J.A., Hand, R.E., Goard, L.J., McAdam, S.P., 1996. Studies on triploid oyster in Australia: Evaluation of cytochalasin B and 6dimethylaminopurine for triploid induction in sidney rock oysters Saccostrea commercialis (Iredale and Roughley). Aquaculture Research 27: 689-698.

80. Nell, J.A., O’Connor, W.A., Hand, R.E., McAdam, S.P., 1995. Hatchery production of diploid and triploid clams, Tapes dorsatus (Lamarck 1818): A potential new species for aquaculture. Aquaculture 130(4): 389-394.

81. Normand, J., Ernande, B., Haure, J., McCombie, H., Boudry, P., 2009. Reproductive effort and growth in Crassostrea gigas: comparison of young diploid and triploid oysters issued natural crosses or chemical induction. Aquatic Biology. 7: 229-241.

82. Palacios, E., Racotta, I., Ibarra, A.M., Ramirez, J.L., Avila, S., 2004. Comparison of biochemical composition and muscle hypertrophy 
associated with the reproductive cycle of diploid and triploid scallop argopecten ventricosus. Journal of Shellfish Research 23 (2): 483+

83. Racotta, I.S., Palacios, E., Ibarra, A.M., Ramirez, J.L., Arcos, F., Erjona, N., 2008. Comparative biochemical composition of ploidy group in the lion.paw scallop (Nodipecten subnodosus Sowerby) supports the physiological hypothesis for the lack of advantage in triploid mollusk growth in food-rich environments. Mar Biol. 153: 1245-1256.

84. Rangel-Dávalos C., Chávez-Villalba, J., 1994. Desarrollo Científico y Tecnológico del Cultivo de Madre Perla y Concha Nácar. Secretaría de Pesca.

85. Racotta, I. S., Ramírez, J.L., Ibarra, A.M., Rodriguez-Jaramillo, M.C., Carreño, D., Palacios, E., 2003. Growth and gametogenesis in the lion-paw scallop Nodipecten (Lyropecten) subnodosus. Aquaculture 217(1-4): 335-349.

86. Ruiz-Verdugo, C.A., Allen, S.K. Jr., Ibarra, A.M., 2001a. Family differences in success of triploid induction and effects of triploidy on fecundity of catarina scallop (Argopecten ventricosus). Aquaculture, 201: 19-33.

87. Ruiz-Verdugo, C.A., Racotta, I.S., Ibarra, A.M., 2001b. Comparative biochemical composition in gonad and adductor muscle of triploid and diploid catarina scallop (Argopecten ventricosus, Sowerby II, 1842)0. J. Exp. Mar. Biol. Ecol., 259: 155-170.

88. Ruiz-Verdugo, C. A., J.L. Ramírez, Allen J.S. Jr., Ibarra A.M., 2000. Triploid catarina scallop (Argopecten ventricosus Sowerby II, 1842): growth and suppression of functional hermaphroditism. Aquaculture, 186:13-32.

89. SAGARPA., 2000. Anuario estadístico de pesca. México D.F. 268 p.

90. Salas, A., García, L., Searcy, R., García, F., Cancino, J.L., Lizárraga, S., 1988. Cultivo extensivo de mejillón azul Mytilus californianus y Mytilus edulis en las costas de Baja California. Informe Anual Tomo I. Cap. 1:1-6 pp. U.A.B.C. - I.I.O. S.E.P.

91. Sevilla, M. 1965. Contribución al conocimiento de la madre perla Pinctada mazatlanica (Hanley, 1854) Rev. Soc. Méx. Hist. Nav. 30: 223-262.

92. Scarpa, J., Toro, J.E., Wada, K.T., 1994. Direct comparison of six methods to induce triploidy in bivalves. Aquaculture, 119: 119-133.

93. Scarpa, J., Wada, K.T., Kamaru, A., 1993. Induction of tetraploidy in Mussels by Suppression of polar body formation. Nippon Suisan Gakkaishi, 59(12): 2017-2023.

94. Shpigel, M., Barber, B.J., Mann, R., 1992. Effects of elevated temperature on growth, gametogenesis, physiology, and biochemical 
composition in diploid and triploid Pacific oysters, Crassostrea gigas Thunberg. J. Exp. Mar. Biol. Ecol., 161: 15-25.

95. Shpigel, M., Spencer, B., 1996. Performance of diploid and triploid Manila clams (Tapes philippinarum, Adams and Reeve) ta various levels of tidal exposure in the UK and in water from fish ponds at Eilat, Israel. Aquaculture 14: 159-171.

96. Stanley, J.G., Allen, S.K. Jr., Hidu, H., 1981. Poliploidy induced in the American oyster Crassostrea virginica with Cytochalasin B. Aquaculture, 12: 1-10

97. Stanley, G.J., Hidu, H., Allen, S.K. Jr., 1984. Growth of American Oysters increased by polyploidy induced by blocking meiosis I but not meiosis II. Aquaculture, 37: 147-155.

98. Stephens, L.B., Downing, S.L., 1988. Inhibiting first polar body formation in Crassostrea gigas produces tetraploids, not meiotic I triploids. Journal of Shellfish Research 7: 550-551.

99. Stephens, L.B., Downing, S.L., 1989. Comparing diploid and triploid gametes from the pacific oyster (Crassostrea gigas) using a scanning electron microscope. Journal of Shellfish Res. 8: 324

100. Stepto, N.K., Cook, P.A., 1998. Induction of triploidy in the South African abalone using Cytochalasin B. Aquaculture International, 6: 161-169.

101. Supan, J.E., Wilson, C.E., Allen, S.K.Jr., 2000. The effect of cytochalasin B dosage on the survival and ploidy of Crassostrea virginica (Gmelin) larvae. Journal of Shellfish Research 19(1): 125128.

102. Suquet, M., Malo, F., Quere, C., Ledu, C., LeGrand, J., Benaddelmouna, A., 2016. Gamete quality in triploid Pacific oysters (Crassostrea gigas). Aquaculture. 451: 11-15.

103. Tabarini, C.L., 1984. Induced triploidy in the bay scallop, Argopecten irradians, and its effect on Growth and Gametogenesis. Aquaculture, 42: 151-160.

104. Thorgaard, G.H., 1983. Chromosome set manipulation and sex control in fish. Pp. 405-434. In Fish Physiology, Vol. 9(B), W. S. Hoard, D.J., Randall, and E.M. Donaldson, eds. Academic Press, London.

105. Toro, J.E., Sanhueza, M.A., Paredes, L., Canello, F., 1995a. Induction of triploid embryos by heat shock in the Chilean northern scallop Argopecten purpuratus Lamarck, 1819. New Zealand J. Mar. and Freshwater Res., 29:101-105.

106. Toro, J.E., Sastre, H., 1995b. Induced triploidy in the Chilean blue mussel, Mytilus chilensis (Hupe, 1854 ), and performance of triploid larvae. J. of Shellfish Res., 14(1): 161-164. 
107. Uchimura, Y., Komaru, A., Wada, K.T., Ieyama, H., Yamaki, M., Furuta, H., 1989. Detection of induced triploidy at different ages for larvae of the japanese pearl oyster, Pinctada fucata martensii, by microfluorometry with DAPI staining. Aquaculture 76: 1-9.

108. Utting, S.D., Child, A.R., 1994. Genetic manipulation of the manila clam (Tapes philippinarum) using cytochalasin B to induce triploidy. Aquaculture 120: 271-282.

109. Utting, S.D., Doyou, J., 1992. the increased utilization of egg lipid reserves following induction of triploidy in the manila clam Tapes philippinarum. Aquaculture 103: 17-28.

110. Utting, S.D., Millican, P.F., Laing, I., 1996. The breeding potential and bichemical composition of triploid Manila clams, Tapes philippinarum Adams and Reeve. Aquaculture research 27: 573-580.

111. Wada, K.T., Komaru, A., Uchimura, Y., 1989. Triploid production in the Japanese pearl oyster Pinctada fucata martensii. Aquaculture 76: 11-19.

112. Yamamoto, S., Sugawara, Y., 1988. Induced triploidy in the mussel Mytilus edulis, by temperatura shock. Aquaculture 72: 21-29.

113. Yang, H., Wang, R., Guo, X., Yu, Y., 1997. Tetraploid induction with Cytochalasin B treatment in scallop Chlamys azumapecten F. 11th International Pectinid Workshop, La Paz, B.C.S., México, 10-15 April 1997. Book of Abstracts, 187-188.

114. Yang, H., Zhang, F.S., Guo, X., 2000. Triploid and tetraploid Zhikong Scallop, Chlamys farreri Jones et preston, Produced by Inhibiting Polar body I. Marine Biotechnology, 2, 466-475.

115. Yang, H., Guo, X., 2004. Tetraploid induction by meiosis inhibition in the dwart surfclam Mulinia lateralis (Say 1822): effects of Cytochalasin B duration. Aquaculture Research 35: 1187-1194.

116. Zhang, G., Wang, Z., Chang, Y., Song, J., Ding, J., Zhao, S., Guo, X., 2000. Tetraploid induction in the Pacific abalone Haliotis discus hannai Ino with 6-DMAP and CB. J. Shellfish Res., 19: 540541. 
Tabla 1. Resumen de experimentos en inducción a la triploidía usando CB, en moluscos.

\begin{tabular}{|c|c|c|c|c|c|c|}
\hline Referencia & Especie & $\begin{array}{l}\text { Concentración } \\
\text { mg/L de CB }\end{array}$ & $\begin{array}{l}\text { Inicio } \\
\text { postfert. } \\
\text { (min) }\end{array}$ & $\begin{array}{l}\text { Durac. } \\
\text { Tratam } \\
\text { (min) }\end{array}$ & Éxito (\%) & $\begin{array}{c}\text { Supervivencia a } \\
\text { larva D (\%) }\end{array}$ \\
\hline $\begin{array}{c}\text { Haliótidos } \\
\text { Stepto y Cook, } \\
1998 \\
\text { Miídos }\end{array}$ & Haliotis midae & 0.5 & 3 & 20 & 70.9 & 15.8 \\
\hline $\begin{array}{c}\text { Allen et al., } \\
1982 \\
\text { Mitílidos }\end{array}$ & Mya arenaria & 1.0 & $\begin{array}{c}0 \\
15\end{array}$ & $\begin{array}{l}15 \\
15\end{array}$ & $\begin{array}{c}84-100 \\
75-80\end{array}$ & $\begin{array}{l}1.4 \\
4.6\end{array}$ \\
\hline $\begin{array}{l}\text { Beaumont y } \\
\text { Kelly, } 1989\end{array}$ & Mytilus edulis & $\begin{array}{l}0.1 \\
0.5 \\
1.0\end{array}$ & $\begin{array}{c}5(\mathrm{M} \mathrm{I}) \\
30(\mathrm{M} \mathrm{II})\end{array}$ & $\begin{array}{l}15 \\
20\end{array}$ & $\begin{array}{l}(0.1,30,15) 38 \\
(0.5,30,15) 60 \\
(1.0,30,15) 50\end{array}$ & $\begin{array}{l}38.7 \\
62.5 \\
48.7\end{array}$ \\
\hline $\begin{array}{c}\text { Scarpa et al., } \\
1994 \\
\text { Ostréidos }\end{array}$ & $\begin{array}{c}\text { Mytilus } \\
\text { galloprovinciallis }\end{array}$ & 1.0 & 20 & 15 & 86 & \\
\hline $\begin{array}{c}\text { Allen y } \\
\text { Bushek, } 1992\end{array}$ & $\begin{array}{l}\text { Crassostrea } \\
\text { virginica }\end{array}$ & 1.0 & $50 \%$ CP1 & 20 & 79 & 22 \\
\hline $\begin{array}{c}\text { Allen y } \\
\text { Downing,1986 }\end{array}$ & $\begin{array}{l}\text { Crassostrea } \\
\text { gigas }\end{array}$ & 1.0 & $30-45$ & 15 & (30) 96.3 & \\
\hline $\begin{array}{c}\text { Barber y } \\
\text { Mann, } 1991\end{array}$ & $\begin{array}{c}\text { Crassostrea } \\
\text { virginica }\end{array}$ & 1.0 & 15 & 15 & 96 & \\
\hline $\begin{array}{c}\text { Barber et al., } \\
1992\end{array}$ & $\begin{array}{c}\text { Crassostrea } \\
\text { virginica }\end{array}$ & $\begin{array}{c}0.1,0.5 \text { y } 1.0 \\
0.25 \text { y } 0.5 \\
0.1 \text { у } 0.25\end{array}$ & $\begin{array}{c}25 \text { y } 20 \\
13 \\
15\end{array}$ & $\begin{array}{c}10 \text { y } 22 \\
15 \\
10\end{array}$ & $\begin{array}{c}(0.25,13,10- \\
15) 96\end{array}$ & 84 \\
\hline $\begin{array}{l}\text { Desrosiers et } \\
\text { al., } 1993\end{array}$ & $\begin{array}{c}\text { Crassostrea } \\
\text { gigas }\end{array}$ & $\begin{array}{l}0.5 \\
1.0\end{array}$ & $\begin{array}{l}20 \\
20\end{array}$ & $\begin{array}{l}20 \\
15\end{array}$ & $\begin{array}{c}94-100 \\
100\end{array}$ & 14 - 3 semanas \\
\hline $\begin{array}{l}\text { Downing y } \\
\text { Allen, } 1987\end{array}$ & $\begin{array}{l}\text { Crassostrea } \\
\text { gigas }\end{array}$ & 1.0 & $\begin{array}{c}0,15,30 \\
45,60,75, \\
90,105 \\
120\end{array}$ & 15 & $(1.0,30,15) 88$ & 56 \\
\hline $\begin{array}{c}\text { Gerard et al., } \\
1999\end{array}$ & $\begin{array}{l}\text { Crassostrea } \\
\text { gigas }\end{array}$ & 1.0 & $\begin{array}{c}5 \\
40\end{array}$ & $\begin{array}{l}10 \\
10\end{array}$ & $\begin{array}{l}16-55 \\
60-95\end{array}$ & \\
\hline $\begin{array}{l}\text { Gerard et al., } \\
\text { 1994b }\end{array}$ & $\begin{array}{c}\text { Crassostrea } \\
\text { gigas }\end{array}$ & 1.0 & 20 & 15 & 95 & 36 \\
\hline $\begin{array}{l}\text { Hawkins et } \\
\text { al., } 1994\end{array}$ & Ostrea edulis & 1.0 & 40 у 90 & 20 & N.A. & \\
\hline $\begin{array}{l}\text { Nell et al., } \\
1994\end{array}$ & $\begin{array}{c}\text { Saccostrea } \\
\text { commercialis }\end{array}$ & 0.5 & 23 & 20 & $\begin{array}{c}81 \\
85 \text { (semilla) }\end{array}$ & \\
\hline $\begin{array}{l}\text { Nell et al., } \\
1996\end{array}$ & $\begin{array}{l}\text { Saccostrea } \\
\text { commercialis }\end{array}$ & $\begin{array}{c}0.75 \\
1.5\end{array}$ & 30 & 15 & 83 & 43 \\
\hline $\begin{array}{c}\text { Stanley et al., } \\
1981\end{array}$ & $\begin{array}{c}\text { Crassostrea } \\
\text { virginica }\end{array}$ & $0.1,1.0$ y 5 & 50 & 20 & & \\
\hline $\begin{array}{c}\text { Stanley et al., } \\
1984\end{array}$ & $\begin{array}{c}\text { Crassostrea } \\
\text { virginica }\end{array}$ & 0.5 & 15 & $\begin{array}{c}0-15 \\
15-30\end{array}$ & $\begin{array}{l}61 \\
72\end{array}$ & \\
\hline $\begin{array}{l}\text { Supan et al., } \\
2000\end{array}$ & $\begin{array}{l}\text { Crassostrea } \\
\text { virginica }\end{array}$ & $\begin{array}{c}0.125 \\
0.25 \\
0.50\end{array}$ & $\begin{array}{l}50 \% \text { CP1 } \\
50 \% \text { CP1 } \\
50 \% \text { CP1 }\end{array}$ & $\begin{array}{l}10 \\
10 \\
10\end{array}$ & $\begin{array}{c}13 \\
61.8 \\
68.2\end{array}$ & \\
\hline $\begin{array}{c}\text { Pectínidos } \\
\text { Baron et al., } \\
1989\end{array}$ & Chlamys varia & 1.0 & $10,20,30$ & 15 & $\begin{array}{c}(1,20 \\
15) 78.5\end{array}$ & 87.5 \\
\hline $\begin{array}{l}\text { Beaumont, } \\
1986\end{array}$ & Pecten maximus & $\begin{array}{l}0.1 \\
0.5\end{array}$ & $\begin{array}{l}10 \\
10\end{array}$ & $\begin{array}{l}20 \\
20\end{array}$ & $\begin{array}{l}23 \\
36\end{array}$ & \\
\hline $\begin{array}{l}\text { Jackson et al., } \\
2002\end{array}$ & $\begin{array}{l}\text { Placopecten } \\
\text { magellanicus }\end{array}$ & $\begin{array}{c}0.25 \\
0.5 \\
0.25 \\
0.5\end{array}$ & & & $\begin{array}{l}13 \\
16 \\
39 \\
65\end{array}$ & \\
\hline
\end{tabular}


Continuación Tabla 1. Resumen de experimentos en inducción a la triploidía usando CB, en moluscos.

\begin{tabular}{|c|c|c|c|c|c|c|}
\hline Referencia & Especie & $\begin{array}{l}\text { Concentrac. } \\
\text { mg/L de CB }\end{array}$ & $\begin{array}{c}\text { Inicio } \\
\text { postfert. } \\
\text { (min) }\end{array}$ & $\begin{array}{l}\text { Durac. } \\
\text { Tratam } \\
\text { (min) }\end{array}$ & Éxito (\%) & $\begin{array}{c}\text { Supervivencia a } \\
\text { larva D (\%) }\end{array}$ \\
\hline \multirow{2}{*}{$\begin{array}{c}\text { Ruiz-Verdugo } \\
\text { et al., } 2000\end{array}$} & Argopecten & 0.1 & $50 \%$ CP1 & 15 & 8 & 15 \\
\hline & ventricosus & 0.5 & $50 \%$ CP1 & 15 & 58 & 9 \\
\hline \multirow{2}{*}{$\begin{array}{l}\text { Ruiz-Verdugo } \\
\text { et al., } 2001\end{array}$} & Argopecten & 0.5 & $30-40 \%$ CP1 & 15 & $0-100$ & $2.06-33.16$ \\
\hline & ventricosus & 0.5 & 30-40\% CP1 & 15 & $36-100$ & $1.76-12.74$ \\
\hline \multirow[t]{2}{*}{ Tabrini, 1984} & Argopecten & 0.05 & 10 & 20 & 66 & 20 \\
\hline & irradians & 0.1 & 10 & 20 & 94 & 6 \\
\hline \multicolumn{7}{|l|}{ Pteridos } \\
\hline $\begin{array}{c}\text { Jiang et al., } \\
1993\end{array}$ & $\begin{array}{l}\text { Pinctada } \\
\text { martensii }\end{array}$ & 0.75 y 1.0 & 2 у 17 & 15 y 17 & & \\
\hline $\begin{array}{c}\text { Uchimura et } \\
\text { al., } 1989\end{array}$ & $\begin{array}{c}\text { Pinctada fucata } \\
\text { martensii }\end{array}$ & 0.5 & 5 & 15 & 65.4 & \\
\hline \multirow[t]{2}{*}{$\begin{array}{c}\text { Wada et al., } \\
\quad 1989\end{array}$} & Pinctada fucata & 0.1 & $\begin{array}{c}5,10,15,20 \\
25,25 \text { у } 30\end{array}$ & 15 & $\begin{array}{c}(0.1,20,15) \\
52\end{array}$ & \\
\hline & & 0.5 & $\begin{array}{c}3,6,9,12,15 \\
18,21,24,27 \\
\text { у } 30\end{array}$ & 15 & $\begin{array}{c}(0.5,20,15) \\
100\end{array}$ & 39 \\
\hline \multicolumn{7}{|l|}{ Venéridos } \\
\hline $\begin{array}{c}\text { Dufy y Diter, } \\
1990\end{array}$ & $\begin{array}{c}\text { Ruditapes } \\
\text { philippinarum }\end{array}$ & 1.0 & 20 & 15 & 75.8 & 19.3 \\
\hline \multirow{10}{*}{$\begin{array}{c}\text { Gerard et al., } \\
\text { 1994a }\end{array}$} & Ruditapes & 0.5 & 10 & 15 & 63 & 52 \\
\hline & decussatus & 0.5 & 15 & 15 & 63 & 35 \\
\hline & & 0.5 & 20 & 15 & 36 & 29 \\
\hline & & 0.5 & 25 & 15 & 3 & - \\
\hline & & 0.5 & 10 & 15 & 50 & 65 \\
\hline & & 0.5 & 15 & 15 & 63 & 68 \\
\hline & & 1.0 & 15 & 15 & 95 & 31 \\
\hline & & 1.0 & 15 & 20 & 94 & 44 \\
\hline & & 1.0 & 15 & 15 & 85 & 26 \\
\hline & & 1.0 & 15 & 20 & 95 & 24 \\
\hline Gosling y & Ruditapes & 0.5 & 15 & 15 & 50 & \\
\hline Nolan, 1989 & decussatus & 1.0 & 15 & 15 & 40 & \\
\hline $\begin{array}{c}\text { Nell et al., } \\
1995\end{array}$ & $\begin{array}{c}\text { Ruditapes } \\
\text { philippinarum }\end{array}$ & 1.0 & & 15 & $56-85$ & \\
\hline Utting y & Ruditapes & 0.5 & 15 & 15 & 70 & 45 \\
\hline Child, 1994 & philippinarum & 0.5 & 20 & 20 & 75 & \\
\hline $\begin{array}{c}\text { Utting y } \\
\text { Doyou, } 1992\end{array}$ & $\begin{array}{c}\text { Ruditapes } \\
\text { philippinarum }\end{array}$ & 0.5 & & & $67-73$ & \\
\hline
\end{tabular}

Tabla 2. Resumen de ventajas de los triploides sobre los diploides en crecimiento en grupos de moluscos.

\begin{tabular}{cccc}
\hline Especie & Tejido & \% incremento en 3N & Referencia \\
\hline Ostréidos & & & \\
\hline Crassostrea gigas & Biomasa húmeda & 81 & Akashige y Fushimi, 1991 \\
Crassostrea gigas & Biomasa húmeda & 70 & Landau y Guo, 1999 \\
Crassostrea virginica & Biomasa húmeda & 29 & Barber y Mann, 1991 \\
Crassostrea virginica & Biomasa húmeda & 60 & Matthiessen y Davis, 1992 \\
Ostrea edulis & Biomasa seca & 61 & Hawkins et al., 1994 \\
Saccostrea commercialis & Biomasa húmeda & 31 & Hand et al., 1998. \\
Saccostrea commercialis & Biomasa húmeda & 41 & Nell et al., 1994 \\
Pectínidos & & & \\
Argopecten irradians & Biomasa húmeda & 36 & Tabarini, 1984 \\
Argopecten ventricosus & Músculo aductor & 73 & \\
& Músculo aductor & 167 & Ruíz-Verdugo et al., 2000 \\
\hline
\end{tabular}




\begin{tabular}{cccc}
\hline Chlamys nobilys & Biomasa húmeda & $32-59$ & Komaru y Wada, 1989 \\
& Biomasa seca & 52 & Yang, et al., 2000 \\
Chlamys farreri & Biomasa húmeda & 81 & \\
Músculo aductor & $44-96$ & Jiang et al., 1991 \\
Ptéridos & & $27-58$ & Guo y Allen, 1994a \\
$\begin{array}{c}\text { Pinctada martensii } \\
\text { Mactrídos }\end{array}$ & Biomasa húmeda & 72 & \\
Mulinia lateralis & Biomasa húmeda & &
\end{tabular}

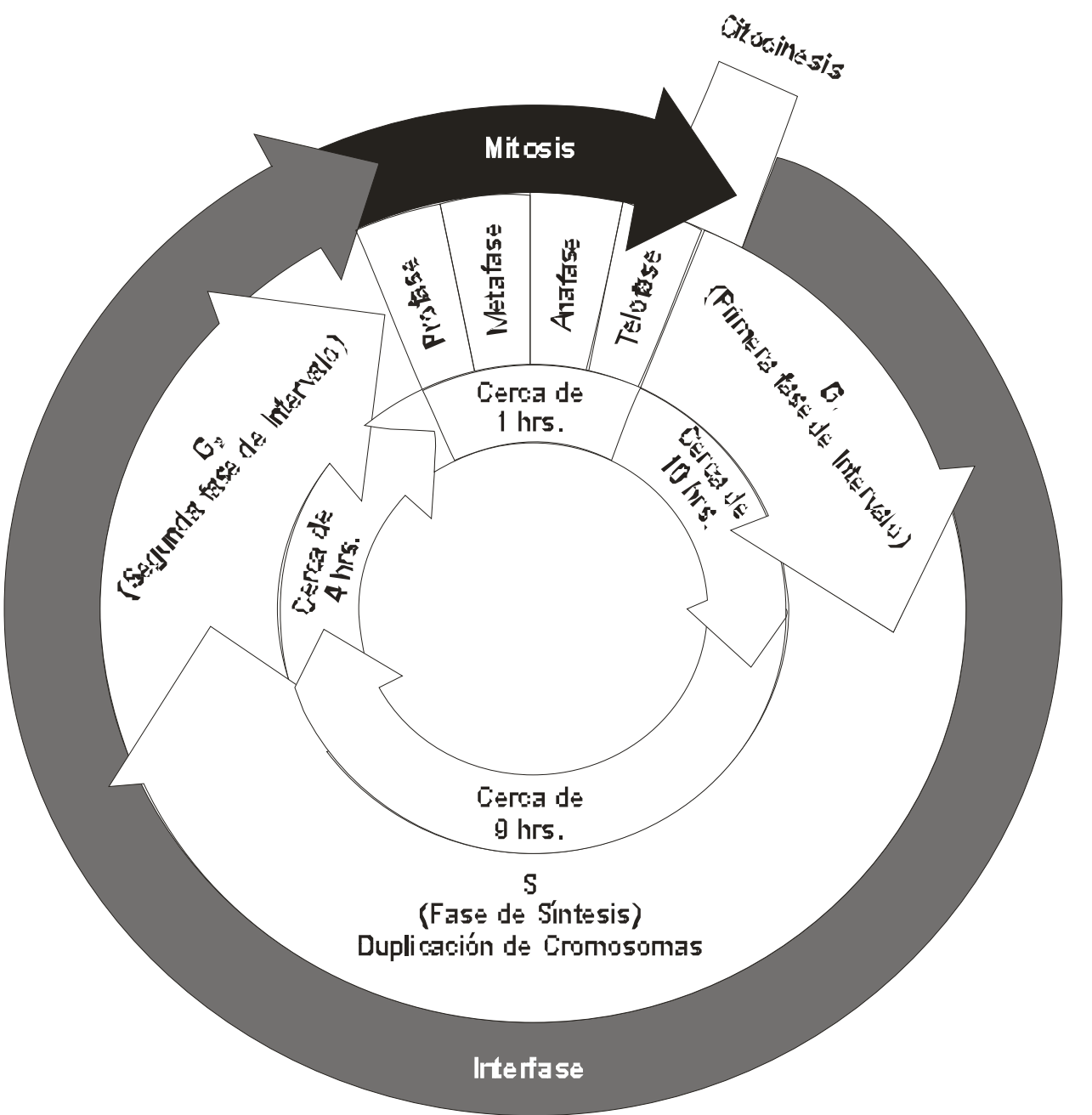

Fig. 1. Este diagrama circular representa un solo ciclo celular. El tiempo de generación, es el lapso necesario para completar un ciclo, comprende la división celular (mitosis y citocinesis) y la interfase. (Tomado de Herskowitz, 1987). 


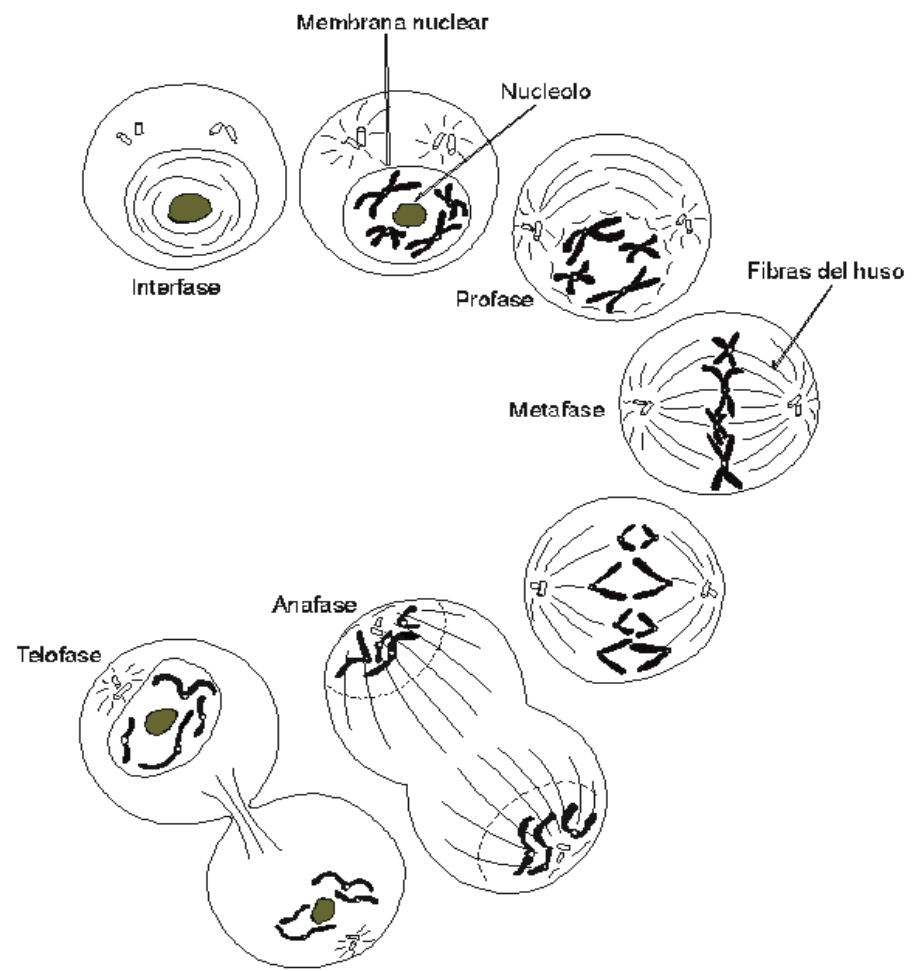

Fig. 2. Esquema de la mitosis. Tomado de Hartl (1991).

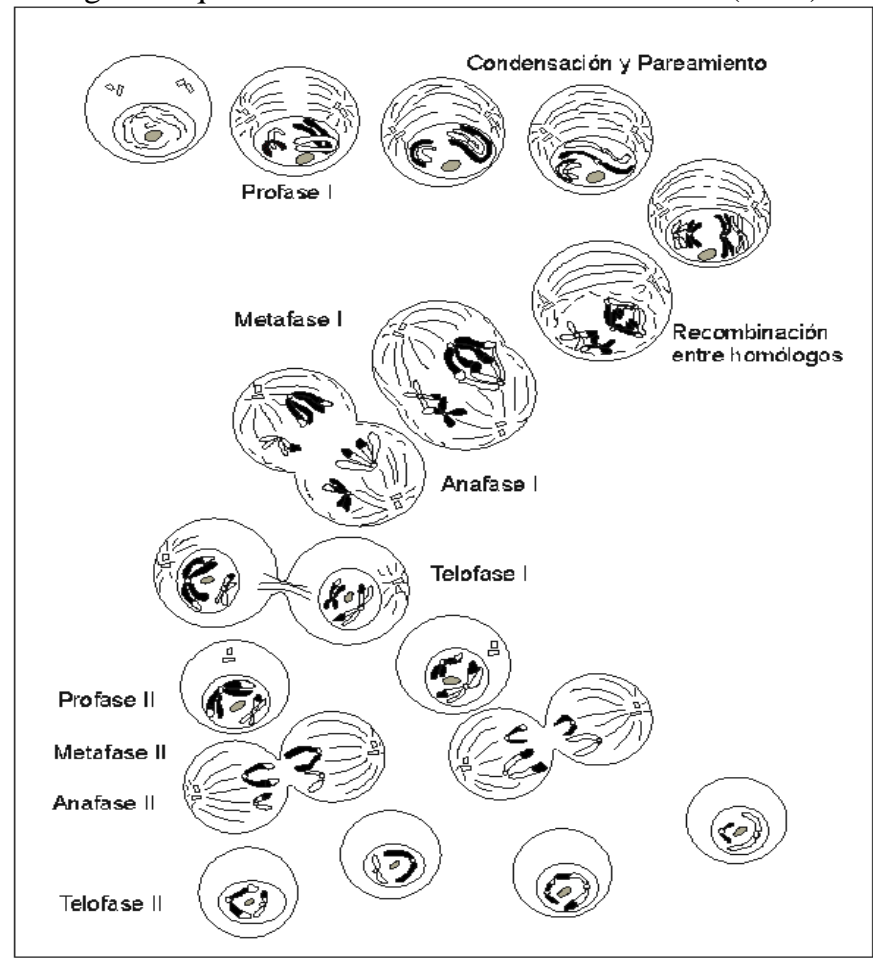

Fig. 3. Esquema de la meiosis. Tomado de Hartl (1991). 


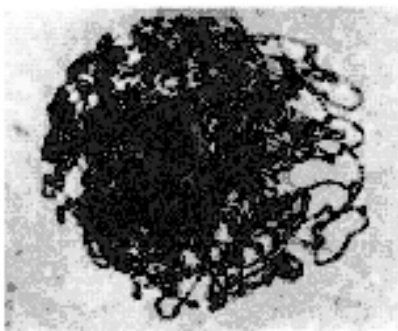

A

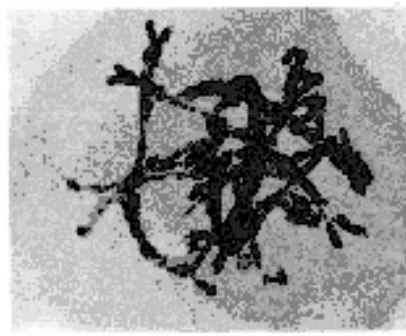

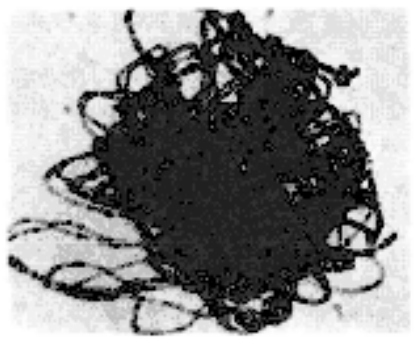

B

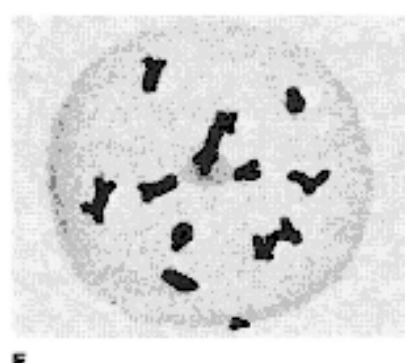

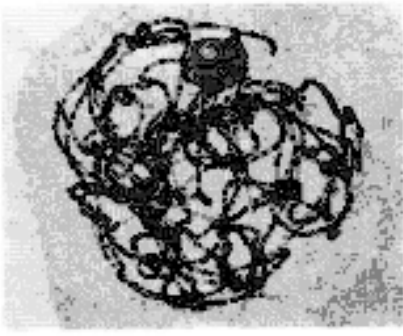

C

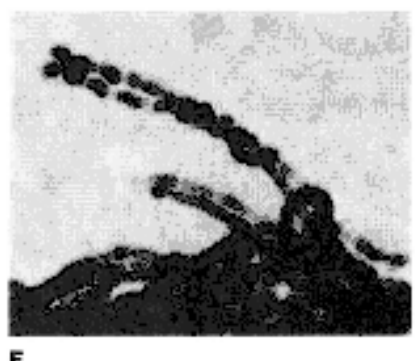

Fig. 3.1 . Subestadios de profase de la primera división meiótica en microsporocitos de un Lily (Lilium longiflorum) A. Leptoteno, en el que la condensación de los cromosomas es iniciada y los cromómeros son visibles a lo largo de la longitud de los cromosomas. B. Cigoteno, en este subestadio ocurre el apareamiento (sinapsis) de los cromosomas homólogos. Regiones pareadas y no pareadas pueden observarse particularmente en la parte baja izquierda de esta fotografía. C. Paquiteno, ocurren los "crossing-over" entre los cromosomas homólogos. D. Diploteno, caracterizado por la repulsión mutua de los cromosomas homólogos pareados que quedan unidos en uno o más puntos (quiasmas) a lo largo de su longitud. E. Diacinesis, en el que los cromosomas alcanzan su máxima contracción. F. Cigoteno, (en magnificación alta en otra célula), mostrando homólogos pareados y emparejamiento de cromosomas durante la sinopsis. [Cortesía de Marta Walters (A,B,C,E,F) y Herbert Stern (D).] Tomada de Hartl (1991).

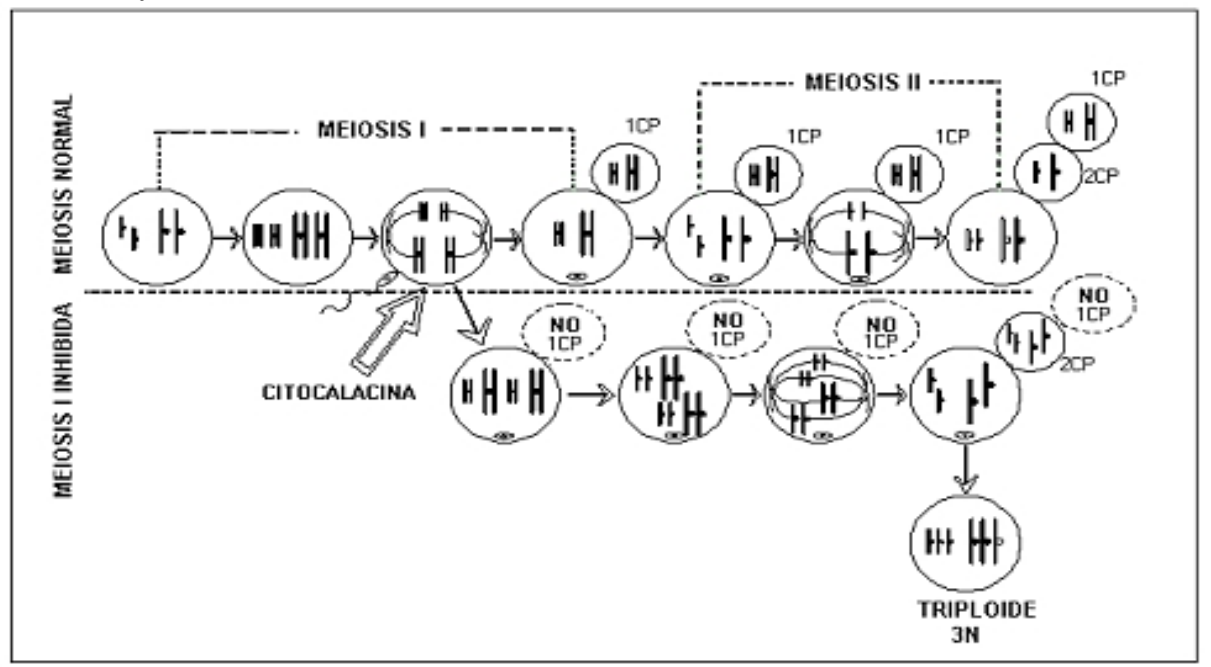

Fig. 4. Representación esquemática de la inhibición del primer cuerpo polar para producir cigotos triploides, en ovocitos normales. Esquema diseñado por A. M. Ibarra. 


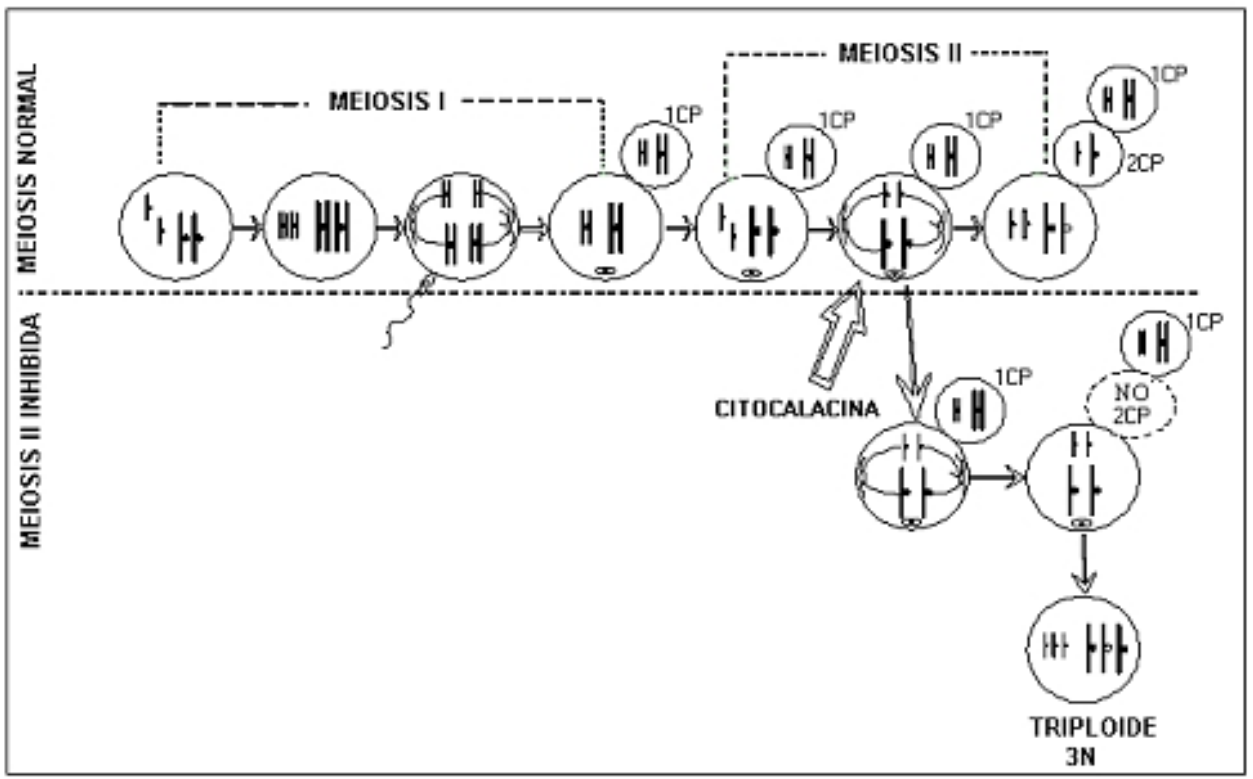

Fig. 5. Representación esquemática de la inhibición del segundo cuerpo polar para producir cigotos triploides, en ovocitos normales. Esquema diseñado por A. M. Ibarra.

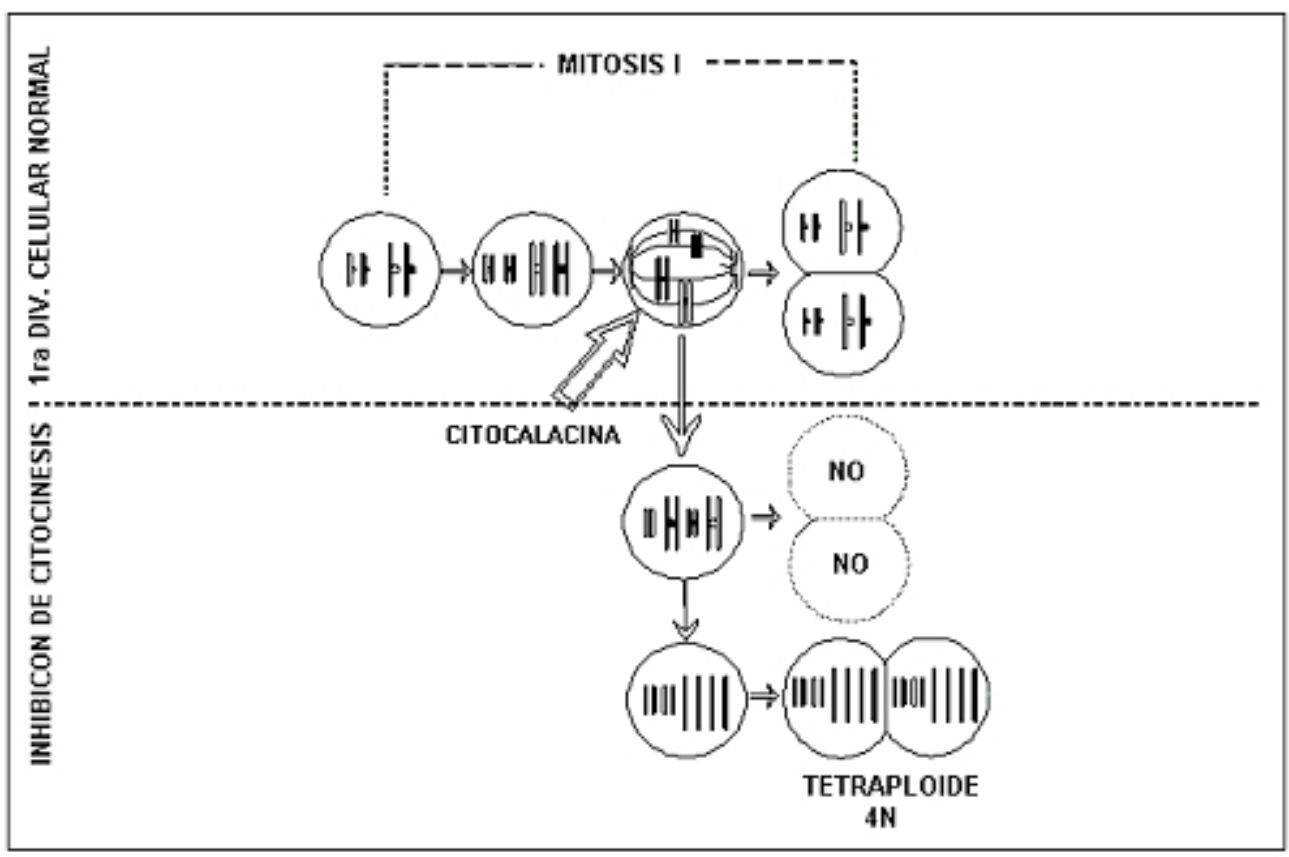

Fig. 6. Representación esquemática de la inhibición de la primera división mitótica, para producir cigotos tetraploides. 


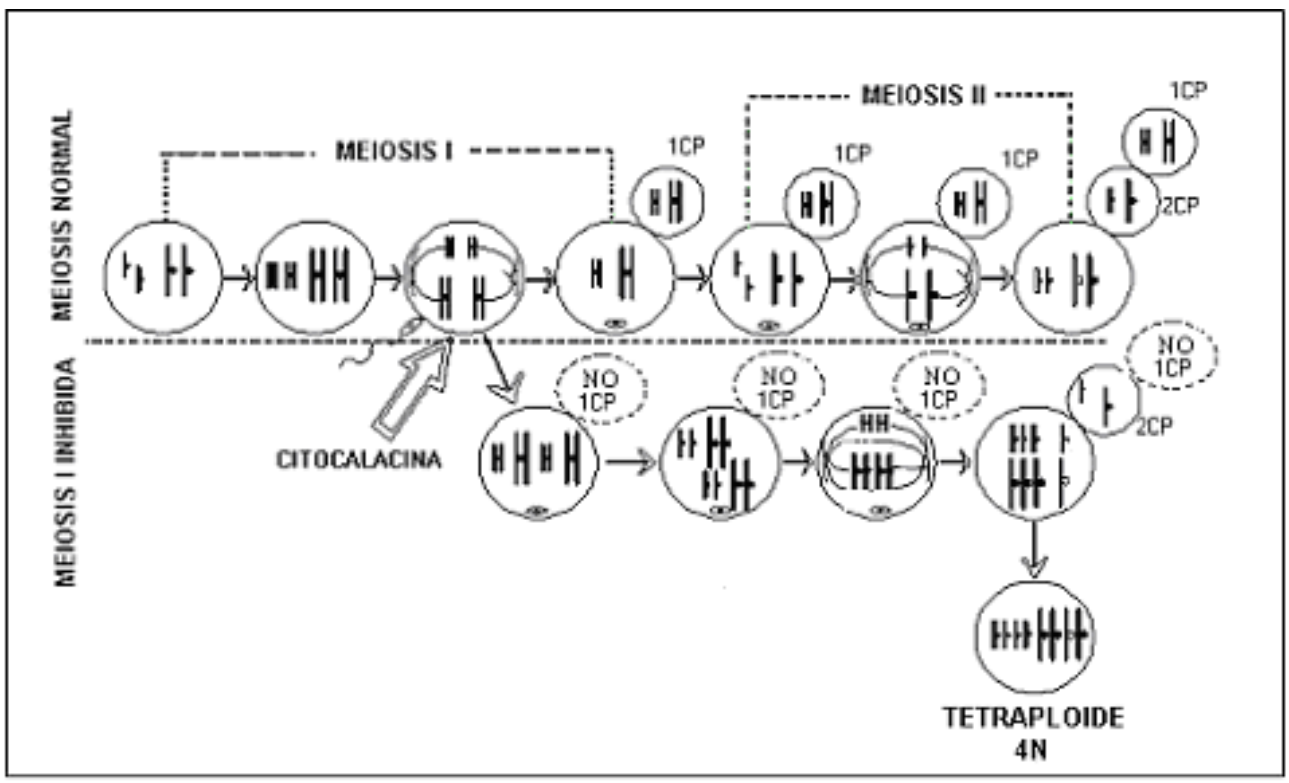

Fig. 7. Representación esquemática de la inhibición del primer cuerpo polar, donde resultan cigotos tetraploides de forma “accidental”. Esquema diseñado por A.M. Ibarra.

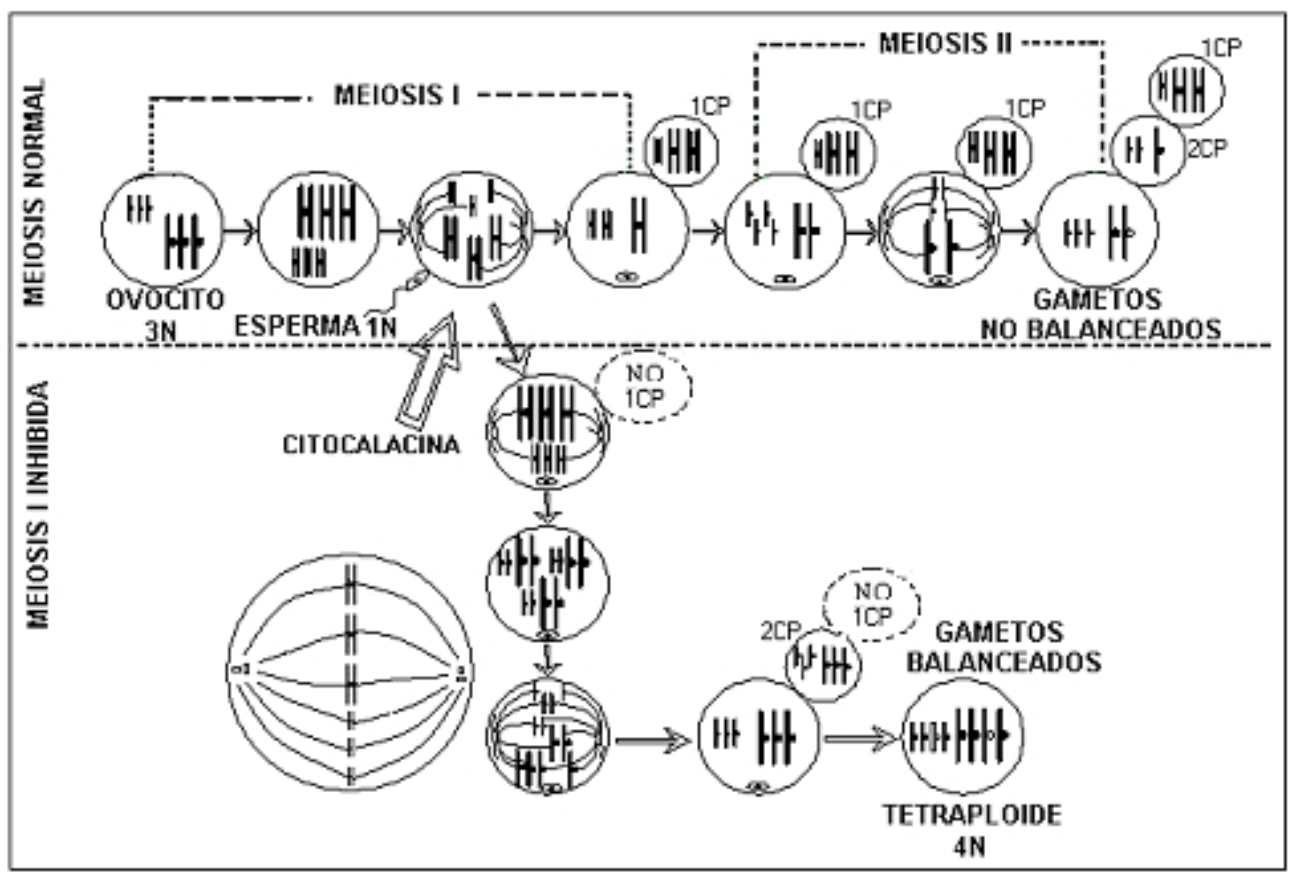

Fig. 8. Representación esquemática de la inhibición del primer cuerpo polar en ovocitos de triploides, fecundados con esperma haploides para producir cigotos tetraploides (Guo y Allen, 1994b), Esquema diseñado por A. M. Ibarra. 